\title{
CALCUL DU SPECTRE D'UNE NILVARIÉTÉ DE RANG DEUX ET APPLICATIONS
}

\author{
HUBERT PESCE
}

\begin{abstract}
RÉsumé. On calcule, en utilisant la théorie des orbites de Kirillov, le spectre d'une nilvariété compacte de rang deux. Puis on utilise ce calcul pour étudier et caractériser les déformations isospectrales de ces variétés.
\end{abstract}

\section{INTRODUCTION}

Soient $(M, g)$ une variété riemannienne compacte et $\Delta$ l'opérateur de Laplace-Beltrami agissant sur $C^{\infty}(M)$ [2]. Cet opérateur possède un spectre discret $\operatorname{Spec}(M, g)$. Il y a peu de variétés riemanniennes dont le spectre est connu de manière explicite (tores plats, bouteilles de Klein plates, sphères munies de leur métrique standard à courbure constante, espaces projectifs [2]). Dans [7], Gordon et Wilson ont calculé le spectre d'une variété d'Heisenberg (i.e., une variété du type $\left(\Gamma \backslash H_{n}, \mathbf{m}\right)$ où $H_{n}$ est le groupe d'Heisenberg de dimension $2 n+1, \Gamma$ un sous-groupe discret cocompact de $H_{n}$ et $\mathbf{m}$ une métrique qui se relève en une métrique invariante à gauche sur $H_{n}$ ). L'idée utilisée est que le Laplacien d'une telle variété s'exprime en fonction de la représentation quasi-régulière de $H_{n}$ dans $L_{\mathbb{C}}^{2}\left(\Gamma \backslash H_{n}\right)$ et que les représentations unitaires irréductibles de $H_{n}$ sont classifiées [17, p. 70].

Le but des deux premières parties de cet article est de calculer le spectre d'une variété du type $(\Gamma \backslash G, \mathbf{m})$ où $G$ est un groupe de Lie simplement connexe nilpotent de rang deux (i.e., le groupe dérivé est inclus dans le centre), $\Gamma$ un sousgroupe discret cocompact de $G$ et $\mathbf{m}$ une métrique qui se relève en une métrique invariante à gauche sur $G$ (i.e. $(\Gamma \backslash G, \mathbf{m})$ est une nilvariété compacte de rang deux).

Dans la première partie, on rappelle le principe de la théorie de Kirillov, théorie dont le but est de décrire les représentations unitaires irréductibles d'un groupe de Lie nilpotent simplement connexe (Proposition I.2).

Dans la deuxième partie, on utilise la théorie de Kirillov pour obtenir, dans le cas où $G$ est nilpotent de rang deux, des formules "explicites" pour les représentations unitaires irréductibles de $G$ et on en déduit le spectre d'une nilvariété compacte de rang deux (Proposition II.1). Un point important pour

Received by the editors July 3, 1991.

1991 Mathematics Subject Classification. Primary 22E25, 22E27, 58G30, 58G40.

Mots-clés. Groupe de Lie nilpotent, théorie de Kirillov, spectre du Laplacien.

Cette recherche a été effectuée en partie dans le cadre du contrat C.E.E. "Gadget" SC1-0105-C au Laboratoire associé au C.N.R.S. no. 188. 
ce calcul est de savoir quelles représentations irréductibles apparaissent dans la représentation quasi-régulière de $G$ dans $L_{\mathbb{C}}^{2}(\Gamma \backslash G)$. Ces représentations sont caractérisées sans hypothèses sur le rang de $G$, on montre cependant que dans le cas où $G$ est nilpotent de rang deux, cette caractérisation prend une forme beaucoup plus explicite; il en est de même pour la multiplicité avec laquelle ces représentations apparaissent (Proposition A.9).

Une question naturelle est de savoir dans quelle mesure le spectre détermine la géométrie de la variété. En particulier, l'égalité $\operatorname{Spec}(M, g)=\operatorname{Spec}\left(M^{\prime}, g^{\prime}\right)$ implique-t-elle que $(M, g)$ et $\left(M^{\prime}, g^{\prime}\right)$ sont isométriques? Milnor a exhibé deux tores plats de dimension 16 isospectraux non isométriques [12]. Depuis, d'autres exemples de caractère sporadique ont été fournis $[9,10,12,20]$. A partir de 1983, Gordon et Wilson [6] puis Sunada [19] ont introduit des méthodes permettant de construire, dans un cadre plus général, des variétés isospectrales. La méthode de Gordon et Wilson a même permis de construire les premiers exemples de déformations isospectrales non triviales en dimension $\geq 2$; voir aussi $[3,6]$ (on appelle déformation isospectrale sur une variété $M$ une famille continue $\left\{g_{t}\right\}$ de métriques sur $M$ telles que les variétés $\left(M, g_{t}\right)$ soient deux à deux isospectrales; une déformation est non triviale si les variétés $\left(M, g_{t}\right)$ ne sont pas deux à isométriques). Les variétés considérées sont du type $(\Gamma \backslash G, \mathbf{m})$ où $G$ est un groupe de Lie nilpotent simplement connexe, $\Gamma$ un sous-groupe discret cocompact de $G$ et $\mathbf{m}$ une métrique qui se relève en une métrique invariante à gauche sur $G$. On définit un sous-groupe de $\operatorname{Aut}(G): \operatorname{AIA}(G, \Gamma)$, le groupe des automorphismes presque intérieurs par rapport à $\Gamma$, qui contient le groupe des automorphismes intérieurs $\operatorname{Inn}(G) . \operatorname{Si} \operatorname{AIA}(G ; \Gamma) \neq \operatorname{Inn}(G)$, on peut construire des déformations isospectrales non triviales de la forme $\left(\Gamma \backslash G, \mathbf{m}_{s}\right)$ avec $\mathbf{m}_{s}=\varphi_{s}^{*} \mathbf{m}$ où $\left\{\varphi_{s}\right\}$ est une famille continue d'éléments de $\operatorname{AIA}(G ; \Gamma)$. Une question naturelle est de savoir si toutes les déformations isospectales sur $\Gamma \backslash G$, par des métriques provenant de métriques invariantes à gauche sur $G$, sont de la forme ci-dessus. Dans [16], il est démontré qui si $G$ est nilpotent de rang deux et non singulier, alors la réponse à la question est positive.

Le but de la troisième partie de cet article est d'utiliser les résultats obtenus dans les deux premières parties pour étudier les déformations isospectrales, par des métriques qui se relèvent en des métriques invariantes à gauche, sur les nilvariétés de rang deux. On démontre tout d'abord que lors d'une telle déformation, la restriction de la métrique au centre du groupe est constante (Proposition III.6). Puis on montre le principal résultat de cette partie, à savoir que toutes les déformations isospectrales sont de la forme décrite ci-dessus (Proposition III.7). Dans un premier Appendice, on fait les calculs utilisés pour décrire le spectre des nilvariétés de rang deux. Dans un deuxième Appendice, on étudie deux cas particuliers, l'un pour lequel $\operatorname{AIA}(G ; \Gamma)=\operatorname{Inn}(G)$, l'autre pour lequel $\operatorname{AIA}(G ; \Gamma) \neq \operatorname{Inn}(G)$, on $y$ redémontre la Proposition III.7 pour ces cas particuliers par des techniques un peu différentes.

Après avoir fini de régider cet article, j'ai appris que Ouyang a obtenu une démonstration différente de la Proposition III.7, et ce en raisonnant par récurrence sur la dimension de la nilvariété [15].

Je remercie C. Gordon et D. De Turck pour les conversations fructueuses que j'ai pu avoir avec eux. 


\section{RAPPELS SUR LA THÉORIE DE KIRILLOV}

Le but de la théorie de Kirillov est de donner une description de l'ensemble des représentations unitaires irréductibles d'un groupe de Lie nilpotent simplement connexe. L'idée de base de la théorie de Kirillov est qu'une représentation unitaire irréductible d'un tel groupe est toujours induite par une représentation de dimension un d'un sous-groupe.

Par la suite $G$ désignera toujours un groupe de Lie nilpotent simplement connexe. On notera $\mathfrak{g}$ son algèbre de Lie, $G^{\prime}=[G, G]$ (resp. $\mathfrak{g}^{\prime}=[\mathfrak{g}, \mathfrak{g}]$ ) le groupe dérivé (resp. l'algèbre dérivée) et on utilisera les notations et conventions suivantes:

- Si $x \in G, I_{x}$ désigne l'automorphisme intérieur défini par $I_{x}(y)=x y x^{-1}$, $(y \in G)$; on notera $\operatorname{Inn}(G)$ le groupe des automorphismes intérieures. Si $X \in$ $\mathfrak{g}, \operatorname{ad}_{X}$ désigne la dérivation intérieure définie par $\operatorname{ad}_{X}(Y)=[X, Y] \quad(Y \in \mathfrak{g})$; on notera $\operatorname{Inn}(\mathfrak{g})$ l'ensemble des dérivations intérieures et $\operatorname{Der}(\mathfrak{g})$ l'ensemble des dérivations de $\mathfrak{g}$.

- Soit $\operatorname{Aut}(G)($ resp. Aut $(\mathfrak{g}))$ le groupe des automorphismes de $G($ resp. $\mathfrak{g})$. L'application $\operatorname{Aut}(G) \rightarrow \operatorname{Aut}(\mathfrak{g}), \varphi \mapsto \varphi_{*} \quad\left(\varphi_{*}\right.$ désigne la différentielle de $\varphi$ en l'élément neutre) est un isomorphisme de groupes (puisque $G$ est simplement connexe) et on identifiera ces deux groupes.

Rappelons, finalement, que l'exponentielle réalise un difféomorphisme de $\mathfrak{g}$ sur $G$ (on notera log son inverse) et que $G$ est unimodulaire [8].

Revenons maintenant à la description des représentations unitaires irréductibles de $G$. Soit $\lambda$ dans $\mathfrak{g}^{*}$, le dual de $\mathfrak{g}$, on dit qu'une sous-algèbre $\mathfrak{h}$ de $\mathfrak{g}$ est subordonnée à $\lambda$ si la restriction de $\lambda$ à l'algèbre dérivée de $\mathfrak{h}$ est nulle (i.e., pour tout $X, Y \in \mathfrak{h}, \lambda([X, Y])=0)$. Une sous-algèbre $\mathfrak{h}$ de $\mathfrak{g}$ est dite subordonnée maximale par rapport à $\lambda$ si $\mathfrak{h}$ est de dimension maximale parmi toutes les sous-algèbres subordonnées à $\lambda$.

Soit $\mathfrak{h}$ une sous-algèbre subordonnée à $\lambda$, on peut construire un caractère $\bar{\lambda}$ sur $H=\exp (\mathfrak{h})$ en posant $\bar{\lambda}(\exp X)=\exp (2 \pi i \lambda(X))$ où $X \in \mathfrak{h}(\bar{\lambda}$ est bien un caractère puisque $\mathfrak{h}$ est subordonnée à $\lambda$ ). A partir d'un tel caractère, on va construire une représentation induite. Pour cela, on a besoin de résultats sur la structure des groupes de Lie nilpotents simplement connexes.

1. Proposition [17]. Soient $G$ un groupe de Lie nilpotent simplement connexe, $\mathfrak{g}$ son algèbre de Lie et $\mathfrak{h}$ une sous-algèbre de $\mathfrak{g}$. Pour toute famille de vecteurs $\left\{X_{1}, \ldots, X_{k}\right\}(k=\operatorname{dim} \mathfrak{g} / \mathfrak{h})$ vérifiant les deux propriétés suivantes:

(1) $\mathfrak{g}=\mathfrak{h} \oplus \mathbb{R} X_{1} \oplus \cdots \oplus \mathbb{R} X_{k}$

(2) $\mathfrak{h}_{i}=\mathfrak{h} \oplus \mathbb{R} X_{1} \oplus \cdots \oplus \mathbb{R} X_{i}$ est une sous-algèbre de $\mathfrak{g}$ pour $i=1, \ldots, k$;

l'application $H \times \mathbb{R}^{k} \rightarrow G,(h, t) \mapsto h \prod_{i=1}^{k} \exp \left(t_{i} X_{i}\right)$ où $t=\left(t_{1}, \ldots, t_{k}\right)$ est un difféomorphisme.

Le difféomorphisme apparaissant dans la proposition précédente induit un difféomorphisme de $\mathbb{R}^{k}$ sur $H \backslash G$ qui permet d'identifier ces deux ensembles. De plus, $G$ opère par translations à droite sur $H \backslash G$ et si l'on identifie $H \backslash G$ et $\mathbb{R}^{k}$, la mesure de Lebesgue est invariante par les translations à droite de $G$ [17]. 
Revenons au problème initial. Soient $\lambda$ dans $\mathfrak{g}^{*}, \mathfrak{h}$ une sous-algèbre subordonnée à $\lambda$ et $H=\exp (\mathfrak{h})$, choisissons $\left\{X_{i}\right\}_{1 \leq i \leq k}(k=\operatorname{dim} \mathfrak{g} / \mathfrak{h})$ comme dans la proposition précédente. Soit $\mathscr{H}_{0}$ l'ensemble des fonctions continues sur $G$ telles que pour $x \in G$ et $h \in H, f(h x)=\bar{\lambda}(h) f(x)$ où $\bar{\lambda}$ est le caractère construit précédemment. D'après la proposition précédente, $\mathscr{H}_{0}$ s'identifie à $C^{0}\left(\mathbb{R}^{k}\right)$, l'ensemble des fonctions continues sur $\mathbb{R}^{k}$, par l'application: $\mathscr{H}_{0} \rightarrow$ $C^{0}\left(\mathbb{R}^{k}\right), f \mapsto \alpha(f)$ où $\alpha(f)$ est définie par

$$
\alpha(f)(t)=f\left(\prod_{i=1}^{k} \exp \left(t_{i} X_{i}\right)\right) \quad \text { si } t=\left(t_{1}, \ldots, t_{k}\right) \in R^{k} .
$$

Considérons maintenant le sous-espace $\mathscr{H}_{1}$ de $\mathscr{H}_{0}$ des éléments $f$ de $\mathscr{H}_{0}$ tels que $\int_{\mathbf{R}^{k}}|\alpha(f)(t)|^{2} d t<+\infty$ et, finalement, désignons par $\mathscr{H}$ le complété de $\mathscr{H}_{1}$ pour la norme $L^{2}\left(\mathscr{H}\right.$ s'identifie à $L_{\mathbb{C}}^{2}\left(\mathbb{R}^{k}\right)$ par l'application $\mathscr{H} \rightarrow L_{\mathbb{C}}^{2}\left(\mathbb{R}^{k}\right)$, $f \mapsto \alpha(f))$. On peut maintenant définir la représentation $\pi(\lambda, H)$ en posant, si $f \in \mathscr{H}$ et $x, y \in G(\pi(\lambda, H)(x) f)(y)=f(y x)$ où $H=\exp (\mathfrak{h})$. La représentation $\pi(\lambda, H)$ s'appelle la représentation induite par $(\lambda, H)$.

On a alors le résultat fondamental suivant [13].

2. Proposition. Soit $G$ un groupe de Lie nilpotent simplement connexe. Avec les notations précédentes:

(i) Si $\lambda$ est dans $\mathfrak{g}^{*}$ et $\mathfrak{h}$ est une sous-algèbre subordonnée à $\lambda, \pi(\lambda, H)$ est une représentation unitaire. Cette représentation est irréductible si et seulement si $\mathfrak{h}$ est une sous-algèbre subordonnée maximale par rapport à $\lambda$. De plus $\pi(\lambda, H)$ ne dépend que de $\lambda$, à équivalence unitaire près, pourvu que $\mathfrak{h}$ soit subordonnée maximale. On peut donc définir $\pi_{\lambda}=\pi(\lambda, H)$ sans ambiguité.

(ii) Pour toute représentation unitaire irréductible $\sigma$ de $G$, il existe $\lambda$ dans $\mathfrak{g}^{*}$ tel que $\sigma$ soit unitairement équivalente à $\pi_{\lambda}$.

(iii) Soient $\lambda$ et $\mu$ dans $\mathfrak{g}^{*}, \pi_{\lambda}$ et $\pi_{\mu}$ sont unitairement équivalentes si et seulement si il existe $x \in G$ tel que $\lambda=\mu \circ\left(I_{x}\right)_{*}$.

(iv) Pour tout $\lambda$ dans $\mathfrak{g}^{*}$ et $\varphi$ dans $\operatorname{Aut}(G), \pi_{\lambda} \circ \varphi$ est unitairement équivalente $\grave{a} \pi_{\lambda \circ \varphi_{*}}$.

Introduisons sur $\mathfrak{g}^{*}$ la relation d'équivalence suivante: $\mu \sim \lambda$ si et seulement si il existe $x \in G$ tel que $\lambda=\mu \circ\left(I_{x}\right)_{*}$ (on notera $\mathscr{O}(\lambda)$ la classe d'équivalence de $\lambda)$. D'apres la proposition précédente, l'ensemble des représentations unitaires irréductibles de $G$ (à équivalence unitaire près) s'identifie à $\mathfrak{g}^{*} / \sim$.

3. Remarque. On retrouve les résultats déjà connus dans le cas où $G=\mathbb{R}^{n}$. En effet, si $\lambda$ et dans $\left(\mathbb{R}^{n}\right)^{*}$, l'unique algèbre subordonnée maximale par rapport à $\lambda$ est $\mathfrak{g}=\mathbb{R}^{n}$ est la représentation $\pi_{\lambda}$ n'est rien d'autre que le caractère $\bar{\lambda}$.

\section{CAlCul DU SPECTRE D'UNe Nilvariété compacte DE RANG DeUX}

Soit $G$ un groupe de Lie nilpotent simplement connexe, un sous-groupe $\Gamma$ de $G$ est dit uniforme si $\Gamma$ est discret et si $\Gamma \backslash G$ est compact; on sait [18] que l'existence d'un tel sous-groupe est équivalente à l'existence d'une base de $\mathfrak{g}$, l'algèbre de Lie de $G$, par rapport à laquelle les constantes de structure sont rationnelles.

Soient $G$ et $\Gamma$ comme ci-dessus et $\mathbf{m}$ une métrique invariante à gauche sur $G$, alors $\Gamma$ opérant par multiplication à gauche sur $G$ comme un groupe 
d'isométries de $(G, \mathbf{m})$, la métrique $\mathbf{m}$ induit une métrique $\overline{\mathbf{m}}$ sur $\Gamma \backslash G$ de sorte que la projection $(G, \mathbf{m}) \rightarrow(\Gamma \backslash G, \overline{\mathbf{m}})$ soit un revêtement riemannien. Par la suite nous identifierons les métriques $\mathbf{m}$ et $\overline{\mathbf{m}}$ et nous nous intéresserons uniquement aux variétés de la forme $(\Gamma \backslash G, \mathbf{m})$ où $\Gamma$ est un sous-groupe uniforme de $G$ et $\mathbf{m}$ est une métrique qui se relève en une métrique invariante à gauche sur $G$.

Le but de cette partie est de calculer le spectre de l'opérateur de LaplaceBeltrami d'une telle variété dans le cas où $G$ est nilpotent de rang deux (i.e., le groupe dérivé est inclus dans le centre) en utilisant la théorie de Kirillov.

Pour cela, on introduit la représentation quasi-régulière $\rho$ de $G$ dans $L_{\mathbb{C}}^{2}(\Gamma \backslash G)$ définie par: $(\rho(x) f)(y)=f(y x)$ où $x, y \in G$ et $f \in L_{\mathbb{C}}^{2}(\Gamma \backslash G)$. Comme tout groupe nilpotent est unimodulaire, cette représentation est bien définie et est unitaire. On définit alors la différentielle de cette représentation comme suit:

$$
\left(\rho_{*}(X)\right) f=\left.\frac{d}{d t} \rho(\exp (t X)) f\right|_{t=0} \quad \text { où } X \in \mathfrak{g} \text { et } f \in C^{\infty}(\Gamma \backslash G) .
$$

$\left(\rho_{*}(X)\right.$ est donc un opérateur différentiel du premier ordre.) On a alors une expression simple de l'opérateur de Laplace-Beltrami $\Delta_{\mathbf{m}}$ de la variété $(\Gamma \backslash G, \mathbf{m})$ [7]:

$$
\Delta_{\mathbf{m}}=-\sum_{i=1}^{n} \rho_{*}\left(X_{i}\right)^{2}
$$

où $\left\{X_{i}\right\}_{1 \leq i \leq n}$ est une base orthonormée quelconque de $\mathfrak{g}$ pour la métrique $\mathbf{m}$ $(n=\operatorname{dim} \mathfrak{g})$.

Comme $\Gamma \backslash G$ est une variété compacte, la représentation quasi-régulière de $G$ dans $L_{\mathbb{C}}^{2}(\Gamma \backslash G)$ se décompose en une somme directe discrète de représentations unitaires irréductibles, chacune apparaissant avec multiplicité finie. Donc, d'après les résultats de la première partie, il existe une partie $\mathscr{A}(\Gamma)$ de $\mathfrak{g}^{*}$ telle que si $\lambda$ et $\mu$ sont dans $\mathscr{A}(\Gamma), \mathscr{O}(\lambda) \cap \mathscr{O}(\mu)=\varnothing$, et pour chaque $\lambda \in \mathscr{A}(\Gamma)$ un entier $m_{\lambda}$ non nul tels que $\rho \sim \sum_{\lambda \in \mathscr{A}(\Gamma)} m_{\lambda} \pi_{\lambda}$ (on explicitera l'ensemble $\mathscr{A}(\Gamma)$ et les entiers $m_{\lambda}$ par la suite). Comme en restriction à chaque espace irréductible $V_{\lambda}(\lambda \in \mathscr{A}(\Gamma)) \rho$ est unitairement équivalente à $\pi_{\lambda}$, la restriction de $\Delta_{\mathbf{m}}$ à $V_{\lambda}$ est unitairement conjuguée à l'opérateur:

$$
\Delta_{\lambda, \mathrm{m}}=-\sum_{i=1}^{n}\left(\pi_{\lambda}\right)_{*}\left(X_{i}\right)^{2}
$$

où $\left\{X_{i}\right\}_{1 \leq i \leq n}$ est une base orthonormée quelconque de $\mathfrak{g}$ pour la métrique et où l'on a posé

$$
\left(\pi_{\lambda}\right)_{*}\left(X_{i}\right)=\left.\frac{d}{d t} \pi_{\lambda}\left(\exp \left(t X_{i}\right)\right)\right|_{t=0} \quad \text { pour } i=1, \ldots, n .
$$

On en déduit que le spectre de la restriction de $\Delta_{\mathbf{m}}$ à $V_{\lambda}$ est égal à celui de $\Delta_{\lambda, \mathbf{m}}$ que l'on notera $\Sigma(\lambda, \mathbf{m})$. D'après ce qui précède, le spectre de $(\Gamma \backslash G, \mathbf{m})$ sera la réunion des $\Sigma(\lambda, \mathbf{m})$, chaque élément de $\Sigma(\lambda, \mathbf{m})$ étant compté avec la multiplicité $m_{\lambda}$. Il ne reste plus qu'à calculer le spectre des opérateurs $\Delta_{\lambda, \mathbf{m}}$.

L'idée est de se ramener à des opérateurs "classiques" dont on connait les fonctions propres (fonctions d'Hermite). Les calculs détaillés sont faits dans l'Appendice I. 
Avant d'énoncer les résultats obtenus, fixons les notations. Si $\lambda$ est dans $\mathfrak{g}^{*}$, on note $B_{\lambda}$ la forme bilinéaire antisymétrique définie par $B_{\lambda}(X, Y)=$ $\lambda([X, Y])$ pour $X, Y$ dans $\mathfrak{g}$ et $\mathfrak{g}_{\lambda}$ le noyau de $B_{\lambda}$. Si $\mathbf{m}$ est une métrique sur $\mathfrak{g}$, on note $u_{\lambda}$ l'opérateur antisymétrique par rapport à $\mathbf{m}$ défini par $B_{\lambda}(X, Y)=\mathbf{m}\left(X, u_{\lambda}(Y)\right)$. Comme $u_{\lambda}$ est antisymétrique, ses valeurs propres sont de la forme $\pm \operatorname{id}_{j}$ pour $j \in\{1, \ldots, k\}$ où $k=\left(\operatorname{dim} \mathfrak{g}-\operatorname{dim} \mathfrak{g}_{\lambda}\right) / 2$ (on suppose que $0<d_{1} \leq d_{2} \leq \cdots \leq d_{k}$ ). Avec ces notations, on montre (Appendice A, p. 446):

- si $\mathfrak{g}_{\lambda}=\mathfrak{g}$, le spectre $\Sigma(\lambda, \mathbf{m})$ de $\Delta_{\lambda, \mathbf{m}}$ est $\Sigma(\lambda, \mathbf{m})=\left\{4 \pi^{2} \sum_{i=1}^{n} \lambda\left(X_{i}\right)^{2}\right\}$ où $\left\{X_{i}\right\}_{1 \leq i \leq n}$ est une base orthonormale quelconque de $\mathfrak{g}$ pour $\mathbf{m}$.

- si $\mathfrak{g}_{\lambda} \neq \mathfrak{g}$, le spectre $\Sigma(\lambda, \mathbf{m})$ de $\Delta_{\lambda, \mathbf{m}}$ est $\Sigma(\lambda, \mathbf{m})=\{\mu(\lambda, p, \mathbf{m}) ; p \in$ $\left.\mathbb{N}^{k}\right\}$ où

$$
\mu(\lambda, p, \mathbf{m})=4 \pi^{2} \sum_{i=1}^{l}\left(\lambda\left(W_{i}\right)\right)^{2}+2 \pi \sum_{j=1}^{k}\left(2 p_{j}+1\right) d_{j}
$$

avec $l=\operatorname{dim} \mathfrak{g}_{\lambda}, k=\left(\operatorname{dim} \mathfrak{g}-\operatorname{dim} \mathfrak{g}_{\lambda}\right) / 2$ et où $\left\{W_{i}\right\}_{1 \leq i \leq l}$ est une base orthonormale de $\mathfrak{g}_{\lambda}$ pour $\mathbf{m}$.

La deuxième étape consiste à caractériser les représentations irréductibles qui apparaissent dans la représentation quasi-régulière de $G$ dans $L_{\mathbb{C}}^{2}(\Gamma \backslash G)$ et à savoir avec quelle multiplicité elles apparaissent. Pour cela, on utilise la caractérisation donnée par Moore [3, p. 42] et on montre que, dans le cas des nilvariétés de rang deux, cette caractérisation prend une forme beaucoup plus explicite. Avant d'énoncer le résultat, remarquons que si $\lambda \in \mathfrak{g}^{*}, B_{\lambda}$ induit une forme bilinéaire antisymétrique $\widetilde{B}_{\lambda}$ non dégénérée sur $\mathfrak{g} / \mathfrak{g}_{\lambda}$ et que, bien que $\log \Gamma$ ne soit pas a priori un réseau, son image $\mathscr{L}_{\lambda}$ dans $\mathfrak{g} / \mathfrak{g}_{\lambda}$ en est un (puisque si $X, Y \in \log \Gamma$, alors $X+Y+\frac{1}{2}[X, Y] \in \log \Gamma$ et $[X, Y] \in \mathfrak{g}^{\prime} \subset \mathfrak{g}_{\lambda}$ ).

Ceci dit, on montre (Appendice A, Proposition 9), que si $G$ est un groupe de Lie nilpotent simplement connexe de rang deux et si $\Gamma$ est un sous-groupe uniforme de $G$, alors la représentation $\pi_{\lambda}$ apparaît dans la représentation quasi-régulière de $G$ dans $L_{\mathbb{C}}^{2}(\Gamma \backslash G)$ si et seulement si $\lambda\left(\log \Gamma \cap \mathfrak{g}_{\lambda}\right) \subset \mathbb{Z}$. La représentation $\pi_{\lambda}$ apparaît alors avec la multiplicité $m_{\lambda}=1$ si $\mathfrak{g}=\mathfrak{g}_{\lambda}$ et $m_{\lambda}=\left(\operatorname{det} \widetilde{B}_{\lambda}\right)^{1 / 2}$ si $\mathfrak{g}_{\lambda} \neq \mathfrak{g}$, le déterminant étant calculé par rapport à n'importe quelle base du réseau $\mathscr{L}_{\lambda}$.

Cela étant, on dit qu'une forme linéaire $\lambda$ est adaptée à $\Gamma$ si $\lambda\left(\log \Gamma \cap \mathfrak{g}_{\lambda}\right) \subset \mathbb{Z}$ et qu'une orbite est adaptée à $\Gamma$ si ses éléments sont adaptés à $\Gamma$.

On a donc montré que si $G$ est un groupe de Lie nilpotent simplement connexe de rang 2 et $\Gamma$ un sous-groupe uniforme de $G$, alors la représentation quasi-régulière $\rho$ de $G$ dans $L_{\mathbb{C}}^{2}(\Gamma \backslash G)$ se décompose comme suit:

$$
\rho \sim \sum_{\lambda \in \mathscr{A}(\Gamma)} m_{\lambda} \pi_{\lambda}
$$

où $\mathscr{A}(\Gamma)$ est une partie quelconque de $\mathfrak{g}^{*}$ dont l'intersection avec toute orbite adaptée à $\Gamma$ se réduit à un élément.

Ecrivons $\mathscr{A}(\Gamma)=\mathscr{A}_{1}(\Gamma) \cup \mathscr{A}_{2}(\Gamma)$ où $\mathscr{A}_{1}(\Gamma)$ est l'ensemble des éléments de $\mathscr{A}(\Gamma)$ qui sont nuls sur $\mathfrak{g}^{\prime}$ (i.e., $\mathfrak{g}_{\lambda}=\mathfrak{g}$ ) et $\mathscr{A}_{2}(\Gamma)$ son complémentaire dans $\mathscr{A}(\Gamma)$. On a alors le résultat suivant.

1. Proposition. Soient $G$ un groupe de Lie nilpotent simplement connexe de rang deux, $\Gamma$ un sous-groupe uniforme de $G$ et $\mathbf{m}$ une métrique invariante à 
gauche sur $G$, alors le spectre $\Sigma(\Gamma \backslash G, \mathbf{m})$ de la variété $(\Gamma \backslash G, \mathbf{m})$ est de la forme $\Sigma(\Gamma \backslash G, \mathbf{m})=\bigcup_{\lambda \in \mathscr{A}(\Gamma)} \Sigma(\lambda, \mathbf{m})$ où l'on a posé:

- si $\lambda \in \mathscr{A}_{1}(\Gamma): \Sigma(\lambda, \mathbf{m})=\left\{4 \pi^{2} \sum_{i=1}^{n} \lambda\left(X_{i}\right)^{2}\right\}$ où $\left\{X_{i}\right\}_{1 \leq i \leq n}$ est une base orthonormée quelconque de $\mathfrak{g}$ pour la métrique $\mathbf{m}$.

- si $\lambda \in \mathscr{A}_{2}(\Gamma): \Sigma(\lambda, \mathbf{m})=\left\{\mu(\lambda, p, \mathbf{m}), p \in \mathbb{N}^{k}\right\}$ où

$$
\mu(\lambda, p, \mathbf{m})=4 \pi^{2} \sum_{i=1}^{l} \lambda\left(X_{i}\right)^{2}+2 \pi \sum_{j=1}^{k}\left(2 p_{j}+1\right) d_{j}
$$

où:

(1) $l=\operatorname{dim} \mathfrak{g}_{\lambda}, k=\left(\operatorname{dim} \mathfrak{g}-\operatorname{dim} \mathfrak{g}_{\lambda}\right) / 2=(n-l) / 2$.

(2) $\left\{X_{i}\right\}_{1 \leq i \leq l}$ est une base orthonormée de $\mathfrak{g}_{\lambda}$ pour la restriction de $\mathbf{m} \grave{a} \mathfrak{g}_{\lambda}$.

(3) $\pm \operatorname{id}_{j}(1 \leq j \leq k)$ sont les valeurs propres non nulles de l'opérateur $u_{\lambda}$ défini par $\mathbf{m}\left(X, u_{\lambda}(Y)\right)=B_{\lambda}(X, Y)$ pour $X, Y \in \mathfrak{g}$. De plus, une valeur propre $\sigma$ apparaît avec une multiplicité $\sum_{\lambda} m_{\lambda}$, la somme portant sur tous les $\lambda \in \mathscr{A}(\Gamma)$ tels que $\sigma \in \Sigma(\lambda, \mathbf{m})$ (les nombres $m_{\lambda}$ sont ceux définis précédemment).

\section{APPLICATION À L'ÉTUDE DES DÉFORMATIONS ISOSPECTRALES}

Le but de cette partie est d'utiliser les résultats obtenus dans la partie II pour étudier les déformations isospectrales, par des métriques invariantes à gauche, sur les variétés du type $\Gamma \backslash G$ où $G$ est une groupe de Lie nilpotent simplement connexe de rang deux et $\Gamma$ est un sous-groupe uniforme de $G$.

Rappelons que les seules déformations isospectrales non triviales connues, en dimension $\geq 2$, sont celles construites par De Turck, Gordon, et Wilson [3, 4, 6]. On va tout d'abord rappeler la principale notion utilisée pour construire ces déformations.

Si $G$ et $\Gamma$ sont comme ci-dessus, on note $\mathscr{L}$ le réseau engendré par log $\Gamma$ et $\mathscr{L}^{*}$ le réseau dual de $\mathscr{L}: \mathscr{L}^{*}=\left\{\lambda \in \mathfrak{g}^{*} \mid \lambda(\mathscr{L}) \subset \mathbb{Z}\right\}$.

1. Proposition $[4,3]$. Soient $G, \Gamma, \mathscr{L}$ et $\mathscr{L}^{*}$ comme précédemment et $\varphi$ un automorphisme de $G$, alors les conditions suivantes sont équivalentes:

(a) Pour tout $\gamma \in \Gamma$, il existe $x \in G$ tel que $\varphi(\gamma)=x \gamma x^{-1}$.

(b) Pour tout $X \in \mathscr{L}$, il existe $x \in G$ tel que $\varphi_{*}(X)=\left(I_{x}\right)_{*}(X)$.

(c) Pour tout $\lambda \in \mathscr{L}^{*}$, il existe $x \in G$ tel que $\lambda \circ \varphi_{*}=\lambda \circ\left(I_{x}\right)_{*}$.

Un automorphisme de $G$ vérifiant une des trois conditions de la proposition précédente est appelée presque intérieur par rapport à $\Gamma$. On note $\operatorname{AIA}(G ; \Gamma)$ l'ensemble des automorphismes presque intérieurs par rapport à $\Gamma$. On a alors le résultat suivant.

2. Proposition $[4,3]$. Si $G, \Gamma, \mathscr{L}$ et $\mathscr{L}^{*}$ sont comme dans la proposition précédente, $\operatorname{AIA}(G ; \Gamma)$ est un groupe de Lie nilpotent simplement connexe dont l'algèbre de Lie est:

$$
\begin{aligned}
\operatorname{AID}(\mathfrak{g} ; \Gamma) & =\{D \in \operatorname{Der}(\mathfrak{g}) \mid \text { pour tout } X \in \mathscr{L}, D(X) \in[\mathfrak{g}, X]\} \\
& =\left\{D \in \operatorname{Der}(\mathfrak{g}) \mid \text { pour tout } \lambda \in \mathscr{L}^{*},\right. \\
& \text { il existe } \left.Y \in \mathfrak{g} \text { tel que } \lambda \circ D=\lambda \circ \operatorname{ad}_{Y}\right\}
\end{aligned}
$$

$(\operatorname{Der}(\mathfrak{g})$ désigne l'ensemble des dérivations de $\mathfrak{g})$.

Dans $[3,6]$ des variétés isospectrales sont construites à l'aide de la notion d'automorphisme presque intérieur relativement à $\Gamma$. La démonstration de 
l'isospectralité des variétés considérées repose uniquement sur les propriétés formelles des représentations $\pi_{\lambda}$ telles qu'elles sont énoncées dans la proposition I.2. On va donner une démonstration qui utilise les calculs faits dans la partie II. De manière plus précise:

3. Proposition. Soient $G$ un groupe de Lie nilpotent, simplement connexe, de rang deux, $\Gamma$ un sous-groupe uniforme de $G, \mathbf{m}$ une métrique invariante à gauche sur $G$ et $\varphi \in \operatorname{AIA}(G ; \Gamma)$, alors les variétés $(\Gamma \backslash G, \mathbf{m})$ et $\left(\Gamma \backslash G, \varphi^{*} \mathbf{m}\right)$ sont isospectrales.

Preuve. On reprend les notations de la partie II. On va montrer que pour tout $\lambda \in \mathscr{A}(\Gamma), \Sigma(\lambda, \mathbf{m})=\Sigma\left(\lambda, \varphi^{*} \mathbf{m}\right)$.

Si $\lambda \in \mathscr{A}_{1}(\Gamma), \Sigma(\lambda, \mathbf{m})=\left\{4 \pi^{2} \sum_{i=1}^{n} \lambda\left(X_{i}\right)^{2}\right\}$ où $\left\{X_{i}\right\}_{1 \leq i \leq n} \quad(n=\operatorname{dim} \mathfrak{g})$ est une base orthonormée de $\mathfrak{g}$ pour $\mathbf{m}$. Puisque $\left\{\varphi_{*}^{-1}\left(X_{i}\right)\right\}_{1 \leq i \leq n}$ est une base orthonormée de $\mathfrak{g}$ pour $\varphi^{*} \mathbf{m}$, il suffit de montrer que $\sum_{i=1}^{n}\left(\lambda \circ \varphi_{*}^{-1}\left(X_{i}\right)\right)^{2}=$ $\sum_{i=1}^{n} \lambda\left(X_{i}\right)^{2}$. Or $\varphi_{*}^{-1}\left(X_{i}\right)=X_{i}+D\left(X_{i}\right)$ pour $i=1, \ldots, n$ où $D \in \operatorname{AID}(\mathfrak{g}, \Gamma)$ et $\lambda \circ D=0$ puisque la restriction de $\lambda$ à $\mathfrak{g}^{\prime}$ est nulle et que $D(\mathfrak{g}) \subset \mathfrak{g}^{\prime}$. On en déduit que $\Sigma(\lambda, \mathbf{m})=\Sigma\left(\lambda, \varphi^{*} \mathbf{m}\right)$.

Supposons maintenant que $\lambda \in \mathscr{A}_{2}(\Gamma)$. Remarquons tout d'abord que, puisque pour tour $X \in \mathfrak{g}^{\prime}, \varphi_{*}(X)=X$ (ceci est vrai puisque l'égalité précédente est vraie pour $X \in \mathscr{L} \cap \mathfrak{g}^{\prime}$ et que $\mathscr{L} \cap \mathfrak{g}^{\prime}$ engendre $\left.\mathfrak{g}^{\prime}[18]\right), B_{\lambda}=B_{\lambda \circ \varphi_{*}}$. On en déduit que $\varphi_{*}^{-1} \mathfrak{g}_{\lambda}=\mathfrak{g}_{\lambda}$, donc si $\left\{X_{i}\right\}_{1 \leq i \leq l}\left(l=\operatorname{dim} \mathfrak{g}_{\lambda}\right)$ est une base orthonormée de $\mathfrak{g}_{\lambda}$ pour $\mathbf{m},\left\{\varphi_{*}^{-1}\left(X_{i}\right)\right\}_{1 \leq i \leq l}$ est une base orthonormée de $\mathfrak{g}_{\lambda}$ pour $\varphi^{*} \mathbf{m}$. On va montrer que $\sum_{i=1}^{l}\left(\lambda \circ \varphi_{*}^{-1}\left(X_{i}\right)\right)^{2}=\sum_{i=1}^{l} \lambda\left(X_{i}\right)^{2}$. Comme $\lambda \circ \varphi_{*}^{-1}=\lambda+\lambda \circ D$ où $D \in \operatorname{AID}(\mathfrak{g} ; \Gamma)$, pour tout $X \in \mathfrak{g}_{\lambda} \cap \mathscr{L}, \lambda \circ \varphi_{*}^{-1}(X)=\lambda(X)$ (puisqu'il existe $Y=Y_{X} \in \mathfrak{g}$ tel que $\left.\lambda \circ D(X)=\lambda([Y, X])=0\right)$. Comme $\mathfrak{g}_{\lambda}$ est une sous-algèbre rationnelle $\left[8\right.$, p. 210], $\mathfrak{g}_{\lambda} \cap \mathscr{L}$ engendre $\mathfrak{g}_{\lambda} ;$ on en déduit que pour tout $X \in \mathfrak{g}_{\lambda}, \lambda \circ \varphi_{*}^{-1}(X)=\lambda(X)$. On a donc bien l'égalité désirée. On va maintenant montrer que si $u_{\lambda}$ et $u_{\lambda}^{\prime}$ sont les deux opérateurs définis par $B_{\lambda}(X, Y)=\mathbf{m}\left(X, u_{\lambda}(Y)\right)=\left(\varphi^{*} \mathbf{m}\right)\left(X, u_{\lambda}^{\prime}(Y)\right)$ par $X, Y \in \mathfrak{g}$, alors $u_{\lambda}$ et $u_{\lambda}^{\prime}$ sont conjugués. Or, comme $B_{\lambda}=B_{\lambda_{\circ} \varphi_{*}}, \mathbf{m}\left(X, u_{\lambda}(Y)\right)=\mathbf{m}\left(\varphi_{*} X, u_{\lambda}\left(\varphi_{*} Y\right)\right)=$ $\left(\varphi^{*} \mathbf{m}\right)\left(X,\left(\varphi_{*}^{-1} u_{\lambda} \varphi_{*}\right)(Y)\right)$ pour $X, Y \in \mathfrak{g}$. On en déduit que $u_{\lambda}^{\prime}=\varphi_{*}^{-1} u_{\lambda} \varphi_{*}$, ce qui montre que ces opérateurs ont mêmes valeurs propres. On peut donc conclure que pour tout $\lambda \in \mathscr{A}(\Gamma)$ et pour tout $p \in \mathbb{N}^{k}\left(k=\left(\operatorname{dim} \mathfrak{g}-\operatorname{dim} \mathfrak{g}_{\lambda}\right) / 2\right)$, $\mu(\lambda, p, \mathbf{m})=\mu\left(\lambda, p, \varphi^{*} \mathbf{m}\right)$, ce qui montre bien que $\Sigma(\lambda, \mathbf{m})=\Sigma\left(\lambda, \varphi^{*} \mathbf{m}\right)$.

4. Remarque. La proposition précédente est vraie sans hypothèses sur le rang de $G$ (i.e., pour tout groupe de Lie nilpotent simplement connexe ayant un sous-groupe uniforme) $[5,6]$.

Il est clair que le groupe des automorphismes intérieurs $\operatorname{Inn}(G)$ est contenu dans $\operatorname{AIA}(G ; \Gamma)$. Cependant si $x \in G$, les variétés $(\Gamma \backslash G, \mathbf{m})$ et $\left(\Gamma \backslash G,\left(I_{x}\right)^{*} \mathbf{m}\right)$ sont isométriques (en effet, la translation à droite $R_{x^{-1}}$ induit une isométrie de $\left(\Gamma \backslash G,\left(I_{x}\right)^{*} \mathbf{m}\right)$ sur $\left.(\Gamma \backslash G, \mathbf{m})\right)$. Pour obtenir des variétés isospectrales non isométriques à l'aide de la proposition précédente, on doit donc choisir $G$ et $\Gamma$ tels que $\operatorname{Inn}(G) \neq \operatorname{AIA}(G ; \Gamma)[5,6]$.

On s'intéresse maintenant au problème des déformations isospectrales sur les variétés du type $\Gamma \backslash G$ où $G$ est un groupe de Lie nilpotent simplement connexe. Remarquons que, une base de $\mathfrak{g}$ étant fixée, l'ensemble des métriques invariantes à gauche s'identifie à l'ensemble des matrices symétriques définies 
positives (on fera souvent cette identification après avoir précisé la base considérée) et sera par la suite muni de la topologie induite par cette identification. Soient donc $\Gamma$ et $G$ comme ci-dessus et soit $\left\{\mathbf{m}_{s}\right\}_{s \in I}$ ( $I$ est un intervalle de $\mathbb{R}$ ou, plus généralement, un espace topologique connexe) une famille continue de métriques invariantes à gauche, on suppose que les variétés $\left(\Gamma \backslash G, \mathbf{m}_{s}\right)$ sont deux à deux isospectrales, que peut-on dire de la famille $\left\{\mathbf{m}_{s}\right\}_{s \in I}$ ?

Par exemple, on montre facilement [21, Théorème 1], que dans la cas où $G$ est abélien, la famille $\left\{\mathbf{m}_{s}\right\}_{s \in I}$ est constante, résultat que l'on utilisera souvent par la suite.

Dans le cas où $G$ est un groupe de Lie nilpotent simplement connexe de rang deux non singulier (i.e., pour tout $X \in \mathfrak{g}-\mathfrak{g}^{\prime}, \operatorname{ad}_{X}(\mathfrak{g})=\mathfrak{g}^{\prime}$ ), les déformations isospectrales sont caractérisées [16].

5. Proposition. Soient $G$ un groupe de Lie nilpotent simplement connexe de rang deux non singulier, $\Gamma$ un sous-groupe uniforme de $G,\left\{\mathbf{m}_{s}\right\}_{s \in I}$ une famille continue de métriques invariantes à gauche. Si les variétés $\left(\Gamma \backslash G, \mathbf{m}_{s}\right)$ sont deux à deux isospectrales, alors il existe une famille continue $\left\{\varphi_{s}\right\}_{s \in I}$ d'éléments de $\operatorname{AIA}(G ; \Gamma)$ telle que pour tout $s \in I, \mathbf{m}_{s}=\varphi_{s}^{*} \mathbf{m}_{s_{0}}\left(s_{0} \in I\right)$.

On peut penser que cette proposition reste vraie si l'on enlève l'hypothèse "non singulier." Dans cette partie on montre, dans un premier temps, que lors d'une déformation isospectrale, la restriction de la métrique au centre du groupe est constante (ce qui est une condition nécessaire pour toutes les déformations isospectrales soient construites à l'aide de la proposition III.3). Ensuite, on montre que la Proposition III.5 reste vraie si l'on enlève l'hypothèse "non singulier."

6. Proposition. Soient $G$ un groupe de Lie nilpotent simplement connexe de rang deux, $\Gamma$ un sous-groupe uniforme de $G$ et $\left\{\mathbf{m}_{s}\right\}_{s \in I}$ une famille continue de métriques invariantes à gauche. Si les variétés $\left(\Gamma \backslash G, \mathbf{m}_{s}\right)$ sont deux à deux isospectrales, alors la restriction de $\mathbf{m}_{s}$ au centre de $G$ est indépendante de $s$.

Preuve. Notons $\mathfrak{z}$ le centre de $\mathfrak{g}$ et $\mathbf{m}_{s}^{\prime}$ la restriction de $\mathbf{m}_{s}$ à $\mathfrak{z}$. Comme $\mathfrak{z}$ est une algèbre de Lie abélienne et que $\log \Gamma \cap \mathfrak{z}$ est un réseau de $\mathfrak{z}$ [18] $\left((\log \Gamma \cap \mathfrak{z}) \backslash \mathfrak{z}, \mathbf{m}_{s}^{\prime}\right)$ est un tore plat et l'application $s \mapsto \mathbf{m}_{s}^{\prime}$ est continue. On va montrer que les tores plats $\left((\log \Gamma \cap \mathfrak{z}) \backslash \mathfrak{z}, \mathbf{m}_{s}^{\prime}\right)$ sont deux à deux isospectraux, ce qui permettra de conclure [21, Théorème 1]. Or le spectre d'un tore plat est bien connu [2]; on définit le réseau dual

$$
(\log \Gamma \cap \mathfrak{z})^{*}=\left\{\lambda \in \mathfrak{z}^{*} \text { tels que } \lambda(\log \Gamma \cap \mathfrak{z}) \subset \mathbb{Z}\right\}
$$

et si $\left\{X_{i}(s)\right\}_{1 \leq i \leq N} \quad\left(N=\operatorname{dim}_{\mathfrak{z}}\right)$ est une base orthonormée quelconque de $\mathfrak{z}$ pour $\mathbf{m}_{s}^{\prime}$, le spectre de $\left((\log \Gamma \cap \mathfrak{z}) \backslash \mathfrak{z}, \mathbf{m}_{s}^{\prime}\right)$ est

$$
\left\{4 \pi^{2} \sum_{i=1}^{N}\left(\lambda\left(X_{i}(s)\right)\right)^{2} ; \lambda \in(\log \Gamma \cap \mathfrak{z})^{*}\right\} .
$$

Il suffit donc de prouver que pour tout $\lambda \in(\mathfrak{z} \cap \log \Gamma)^{*}, \sum_{i=1}^{N}\left(\lambda\left(X_{i}(s)\right)\right)^{2}=$ $\sum_{i=1}^{N}\left(\lambda\left(X_{i}\left(s_{0}\right)\right)\right)^{2}$ où $\left\{X_{i}(s)\right\}_{1 \leq i \leq N}$ est une base orthonormée de $\mathfrak{z}$ où $s_{0}$ est un élément de $I$ fixé. Or, d'après le résultats de la partie II, si $\lambda$ est adaptée à $\Gamma$ 
et si la restriction de $\lambda$ à $\mathfrak{g}^{\prime}$ est non nulle, alors

$$
\mu(\lambda, p, \mathbf{m})=4 \pi^{2} \sum_{i=1}^{l}\left(\lambda\left(Y_{i}\right)\right)^{2}+2 \pi \sum_{j=1}^{k}\left(2 p_{j}+1\right) d_{j}
$$

(où $l=\operatorname{dim} \mathfrak{g}_{\lambda}, k=(\operatorname{dim} \mathfrak{g}-l) / 2$ et $\left\{Y_{i}\right\}_{1 \leq i \leq l}$ est une base orthonormée de $\mathfrak{g}_{\lambda}$ pour $\mathbf{m})$ est dans le spectre de $(\Gamma \backslash G, \mathbf{m})$. Notons $\Sigma$ le spectre commun aux variétés $\left(\Gamma \backslash G, \mathbf{m}_{s}\right)$, alors l'application $I \rightarrow \Sigma, s \mapsto \mu\left(\lambda, p, \mathbf{m}_{s}\right)$ est continue, donc constante, puisque $I$ est connexe et $\Sigma$ est discret. Donc, pour tout $p \in \mathbb{N}^{k}$ et pour tout $s \in I, \mu\left(\lambda, p, \mathbf{m}_{s}\right)=\mu\left(\lambda, p, \mathbf{m}_{s_{0}}\right)$. On vérifie facilement que pour tout $s \in I, \sum_{i=1}^{l}\left(\lambda\left(Y_{i}(s)\right)\right)^{2}=\sum_{i=1}^{l}\left(\lambda\left(Y_{i}\left(s_{0}\right)\right)\right)^{2}$ où $\left\{Y_{i}(s)\right\}_{1 \leq i \leq l}$ est une base orthonormée de $\mathfrak{g}_{\lambda}$ pour $\mathbf{m}_{s}$.

Soit $\lambda \in(\log \Gamma \cap \mathfrak{z})^{*}$, comme $\log \Gamma \cap \mathfrak{z}$ est un sous-réseau du réseau engendré par $\log \Gamma$, quitte à changer $\lambda$ en $a \cdot \lambda$ où $a \in \mathbb{Z}$, on peut supposer que $\lambda$ s'étend en un élément $\tilde{\lambda}$ de $\mathfrak{g}^{*}$ tel que $\tilde{\lambda}(\log \Gamma) \subset \mathbb{Z}$; en particulier $\tilde{\lambda}$ est adapté à $\Gamma$. Supposons que $\mathfrak{g}_{\tilde{\lambda}}=\mathfrak{z}$, alors d'après ce que l'on vient de voir, $\sum_{i=1}^{N}\left(\lambda\left(X_{i}(s)\right)\right)^{2}=$ $\sum_{i=1}^{N}\left(\lambda\left(X_{i}\left(s_{0}\right)\right)\right)^{2}$ où $\left\{X_{i}(s)\right\}_{1 \leq i \leq N}$ est une base orthonormée de $\mathfrak{g}_{\tilde{\lambda}}=\mathfrak{z}$ pour $\mathbf{m}_{s}^{\prime}$ et ce pour tout $s \in I$. Supposons maintenant $\mathfrak{g}_{\dot{\lambda}} \neq \mathfrak{z}$. Comme $\mathfrak{g}_{\dot{\lambda}}$ est une sous-algèbre rationnelle de $\mathfrak{g}[8], \mathfrak{g}_{\mathfrak{\lambda}} \cap \mathscr{L} \quad(\mathscr{L}$ désigne le réseau engendré par $\log \Gamma$ ) est un réseau de $\mathfrak{g}_{\mathfrak{\lambda}}$ et $\log \Gamma \cap_{\mathfrak{z}}$ est un sous-réseau de $\mathfrak{g}_{\mathfrak{\lambda}} \cap \mathscr{L}$. Il existe donc une base $\left\{U_{1}, \ldots, U_{N}, V_{1}, \ldots, V_{N^{\prime}}\right\} \quad\left(N=\operatorname{dim} \mathfrak{z}, N+N^{\prime}=\operatorname{dim} \mathfrak{g}_{\tilde{\lambda}}\right)$ et des entiers $u_{1}, \ldots, u_{N}$ tels que $\left\{u_{1} U_{1}, \ldots, u_{N} U_{N}\right\}$ soit une base de $\log \Gamma \cap \mathfrak{z}$. Soit $\nu \in\left(\mathbb{Z} V_{1} \oplus \cdots \oplus \mathbb{Z} V_{N^{\prime}}\right)^{*} \simeq\left(\mathbb{Z}^{N^{\prime}}\right)^{*}$, alors $\nu+\tilde{\lambda}$ et $\tilde{\lambda}$ coïncident sur $\mathfrak{z}$, donc sur $\mathfrak{g}^{\prime}$. On en déduit que $B_{\tilde{\lambda}_{+\nu}}=B_{\tilde{\lambda}}$, puis $\mathfrak{g}_{\tilde{\lambda}+\nu}=\mathfrak{g}_{\tilde{\lambda}}$. Soit $\left\{X_{i}(s)\right\}_{1 \leq i \leq N}$ une base orthonormée de $\mathfrak{z}$ pour $\mathbf{m}_{s}^{\prime}$; complétons-la en une base orthonormée de $\mathfrak{g}_{\tilde{\lambda}}$ pour $\mathbf{m}_{s}$ avec $\left\{Y_{i}(s)\right\}_{N+1 \leq i \leq N+N^{\prime}}$ et écrivons $Y_{i}(s)=Z_{i}(s)+X_{i}^{\prime}(s)$ où $Z_{i}(s) \in \mathfrak{z}$ et $X_{i}^{\prime}(s) \in \mathbb{R} V_{1} \oplus \cdots \oplus \mathbb{R} V_{N^{\prime}}$ pour $N+1 \leq i \leq N+N^{\prime}$. Pour tout $\nu \in\left(\mathbb{Z}^{N^{\prime}}\right)^{*}, \tilde{\lambda}+\nu$ est adapté à $\Gamma$, donc la quantité $\sum_{i=1}^{N}\left((\tilde{\lambda}+\nu)\left(Y_{i}(s)\right)\right)^{2}$ est indépendante de $s$. On en déduit que les trois égalités suivantes sont vraies pour tout $s \in I$ :

(i)

(ii)

$$
\sum_{j=N+1}^{N+N^{\prime}}\left(\nu\left(X_{j}^{\prime}(s)\right)\right)^{2}=\sum_{j=N+1}^{N+N^{\prime}}\left(\nu\left(X_{j}^{\prime}\left(s_{0}\right)\right)\right)^{2}
$$

(iii)

$$
\sum_{j=N+1}^{N=N^{\prime}} \nu\left(X_{j}^{\prime}(s)\right) \tilde{\lambda}\left(Y_{j}(s)\right)=\sum_{j=N+1}^{N+N^{\prime}} \nu\left(X_{j}^{\prime}\left(s_{0}\right)\right) \tilde{\lambda}\left(Y_{j}\left(s_{0}\right)\right)
$$

$$
\sum_{j=1}^{N}\left(\lambda\left(X_{j}(s)\right)\right)^{2}+\sum_{j=N+1}^{N+N^{\prime}}\left(\tilde{\lambda}\left(Y_{j}(s)\right)\right)^{2}=\sum_{j=1}^{N}\left(\lambda\left(X_{j}\left(s_{0}\right)\right)\right)^{2}+\sum_{j=N+1}^{N+N^{\prime}}\left(\tilde{\lambda}\left(Y_{j}\left(s_{0}\right)\right)\right)^{2}
$$

Par la suite, on utilisera pour faire des calculs matriciels la base $\mathscr{B}=$ $\left\{U_{1}, \ldots, U_{N}, V_{1}, \ldots, V_{N^{\prime}}\right\}$ précédemment citée. Soit $P(s)$ la matrice dont les colonnes sont les coordonnées de $\left\{X_{1}(s), \ldots, X_{N}(s), Y_{N+1}(s), \ldots, Y_{N+N^{\prime}}(s)\right\}$ dans la base $\mathscr{B}$, on peut donc écrire $P(s)$ sous la forme

$$
\left(\begin{array}{c|c}
P_{1}(s) & P_{2}(s) \\
\hline 0 & P_{3}(s)
\end{array}\right)
$$


où $P_{1}(s)$ est une matrice carrée d'orde $N, P_{3}(s)$ une matrice carrée d'ordre $N^{\prime}$ et $P_{2}(s)$ une matrice à $N$ lignes et $N^{\prime}$ colonnes. Enfin, notons $\lambda_{0}$ la restriction de $\tilde{\lambda}$ à $\mathbb{R} V_{1} \oplus \cdots \oplus \mathbb{R} V_{N^{\prime}}$.

Conséquence de (i). Pour tout $\nu \in\left(\mathbb{Z}^{N^{\prime}}\right)^{*}, \nu P_{3}(s)^{t} P_{3}(s)^{t} \nu=\nu P_{3}\left(s_{0}\right)^{t} P_{3}\left(s_{0}\right)^{t} \nu$. On en déduit [21, théorème 1] que pour tous $s \in I, P_{3}(s)^{t} P_{3}(s)=P_{3}\left(s_{0}\right)^{t} P_{3}\left(s_{0}\right)$, donc $P_{3}(s)=P_{3}\left(s_{0}\right) \mathscr{O}(s)$ où $\mathscr{O}(s)$ est une matrice orthoganale d'ordre $N^{\prime}$.

Conséquence de (ii) Pour tout $\nu \in\left(\mathbb{Z}^{N^{\prime}}\right)^{*}$

$$
\sum_{j=N+1}^{N+N^{\prime}} \nu\left(X_{j}^{\prime}(s)\right) \tilde{\lambda}\left(Y_{j}(s)\right)=\sum_{j=N+1}^{N+N^{\prime}} \nu\left(X_{j}^{\prime}\left(s_{0}\right)\right) \tilde{\lambda}\left(Y_{j}\left(s_{0}\right)\right) .
$$

Or, d'après (i), $\nu P_{3}(s)^{t} P_{3}(s)^{t} \lambda_{0}=\nu P_{3}\left(s_{0}\right)^{t} P_{3}\left(s_{0}\right)^{t} \lambda_{0}$ donc

$$
\sum_{j=N+1}^{N+N^{\prime}} \nu\left(X_{j}^{\prime}\left(s_{0}\right)\right) \lambda_{0}\left(X_{j}^{\prime}\left(s_{0}\right)\right)=\sum_{j=N+1}^{N+N^{\prime}} \nu\left(X_{j}^{\prime}(s)\right) \lambda_{0}\left(X_{j}^{\prime}(s)\right) .
$$

On en déduit que

$$
\sum_{j=N+1}^{N+N^{\prime}} \nu\left(X_{j}^{\prime}(s)\right) \lambda\left(Z_{j}(s)\right)=\sum_{j=N+1}^{N+N^{\prime}} \nu\left(X_{j}^{\prime}\left(s_{0}\right)\right) \lambda\left(Z_{j}\left(s_{0}\right)\right),
$$

ce qui est équivalent à

$$
\nu P_{3}(s)^{t} P_{2}(s)^{t} \lambda=\nu P_{3}\left(s_{0}\right)^{t} P_{2}\left(s_{0}\right)^{t} \lambda .
$$

Cette égalité étant vraie pour tout $\nu \in\left(\mathbb{Z}^{N^{\prime}}\right)^{*}$, on en déduit que $P_{3}(s)^{t} P_{2}(s)^{t} \lambda=$ $P_{3}\left(s_{0}\right)^{t} P_{2}\left(s_{0}\right)^{t} \lambda$. D'où, en tenant compte du fait que $P_{3}(s)=P_{3}\left(s_{0}\right) \mathscr{O}(s)$, on obtient $\lambda P_{2}(s)=\lambda P_{2}\left(s_{0}\right) \mathscr{O}(s)$.

Conséquence de (iii). Remarquons que, d'après (i),

$$
\sum_{j=N+1}^{N+N^{\prime}}\left(\lambda_{0}\left(X_{i}^{\prime}(s)\right)\right)^{2}=\sum_{j=N+1}^{N+N^{\prime}}\left(\lambda_{0}\left(X_{i}^{\prime}\left(s_{0}\right)\right)\right)^{2}
$$

puisque $P_{3}(s)^{t} P_{3}(s)=P_{3}\left(s_{0}\right)^{t} P_{3}\left(s_{0}\right)$. D'autre part puisque, d'après (i) et (ii), $\lambda P_{2}(s)^{t} P_{3}(s)^{t} \lambda_{0}=\lambda P_{2}\left(s_{0}\right)^{t} P_{3}\left(s_{0}\right)^{t} \lambda_{0}$, on a

$$
\sum_{j=N+1}^{N+N^{\prime}} \lambda\left(Z_{j}(s)\right) \lambda_{0}\left(X_{j}^{\prime}(s)\right)=\sum_{j=N+1}^{N+N^{\prime}} \lambda\left(Z_{j}\left(s_{0}\right)\right) \lambda_{0}\left(X_{j}^{\prime}\left(s_{0}\right)\right) .
$$

De même, on montre en utilisant (ii) que

$$
\sum_{j=N+1}^{N+N^{\prime}}\left(\lambda\left(Z_{i}(s)\right)\right)^{2}=\sum_{j=N+1}^{N+N^{\prime}}\left(\lambda\left(Z_{i}\left(s_{0}\right)\right)\right)^{2} .
$$

On en déduit que

$$
\sum_{j=N+1}^{N+N^{\prime}}\left(\tilde{\lambda}\left(Y_{j}(s)\right)\right)^{2}=\sum_{j=N+1}^{N+N^{\prime}}\left(\tilde{\lambda}\left(Y_{j}\left(s_{0}\right)\right)\right)^{2}
$$


d'où, en utilisant (iii),

$$
\sum_{i=1}^{N}\left(\lambda\left(X_{i}(s)\right)\right)^{2}=\sum_{i=1}^{N}\left(\lambda\left(X_{i}(s)\right)\right)^{2} .
$$

On a donc bien montré le résultat voulu puisque $\left\{X_{i}(s)\right\}_{1 \leq i \leq N}$ est une base orthonormée de $\mathbf{z}$ pour $\mathbf{m}_{s}^{\prime}$.

En utilisant la proposition précédente, on va démontrer la principal résultat de cette partie qui constitue une généralisation de la Proposition III.5.

7. Proposition. Soient $G$ un groupe de Lie nilpotent de rang deux simplement connexe, $\Gamma$ un sous-groupe uniforme de $G$ et $\left\{\mathbf{m}_{s}\right\}_{s \in I}$ une famille continue de métriques invariantes à gauche. Si les variétés $\left(\Gamma \backslash G, \mathbf{m}_{s}\right)$ sont deux à deux isospectrales, alors il existe une famille continue $\left\{\psi_{s}\right\}_{s \in I}$ d'éléments de $\operatorname{AIA}(G ; \Gamma)$ telle que pour tout $s \in I, \mathbf{m}_{s}=\psi_{s}^{*} \mathbf{m}_{s_{0}},\left(s_{0} \in I\right)$.

Avant de donner la preuve de cette proposition, on va donner une nouvelle caractérisation de $\operatorname{AID}(\mathfrak{g} ; \Gamma)$.

8. Lemme. Soient $G$ et $\Gamma$ comme ci-dessus, alors une dérivation $D$ de $\mathfrak{g}$ est dans $\operatorname{AID}(\mathfrak{g} ; \Gamma)$ si et seulement si pour tout $\lambda \in \mathscr{L}^{*}, D\left(\mathfrak{g}_{\lambda}\right) \subset \operatorname{Ker} \lambda \cap \mathfrak{g}_{\lambda}$.

Preuve. On va utiliser le fait qu'une dérivation $D$ de $\mathfrak{g}$ est dans $\operatorname{AID}(\mathfrak{g} ; \Gamma)$ si et seulement si pour tout $\lambda \in \mathscr{L}^{*}$, il existe $X \in \mathfrak{g}$ tel que $\lambda \circ D=\lambda \circ \operatorname{ad}_{X}$.

Soient donc $D \in \operatorname{AID}(\mathfrak{g}, \Gamma), \lambda \in \mathscr{L}^{*}$ et $X \in \mathfrak{g}$ tels que $\lambda \circ D=\lambda \circ \operatorname{ad}_{X}$, alors pour tout $Y \in \mathfrak{g}_{\lambda}, \lambda \circ D(Y)=\lambda([X, Y])=0$ donc $D\left(\mathfrak{g}_{\lambda}\right) \subset \operatorname{Ker} \lambda$. Comme $D(\mathfrak{g}) \subset \mathfrak{g}^{\prime} \subset \mathfrak{g}_{\lambda}$, on a forcément $D\left(\mathfrak{g}_{\lambda}\right) \subset \operatorname{Ker} \lambda \cap \mathfrak{g}_{\lambda}$.

Réciproquement, soit $D \in \operatorname{Der}(\mathfrak{g})$ tel que pour tout $\lambda \in \mathscr{L}^{*}, D\left(\mathfrak{g}_{\lambda}\right) \subset$ $\left.\operatorname{Ker} \lambda \cap \mathfrak{g}_{\lambda}\right)$. Soit $\lambda \in \mathscr{L}^{*}$, posons $\mu=\lambda+\lambda \circ D$. Alors $\mu$ et $\lambda$ coïncident sur $\mathfrak{g}_{\lambda}$, on va voir que cette condition implique qu'il existe $X \in \mathfrak{g}$ tel que $\lambda \circ D=$ $\lambda \circ \operatorname{ad}_{X}$. En effet, il existe une base $\left\{X_{1}, \ldots, X_{k}, Y_{1}, \ldots, Y_{k}, Z_{1}, \ldots, Z_{l}\right\}$ de $\mathfrak{g}(2 k+l=\operatorname{dim} \mathfrak{g})$ et des réels non nuls $r_{1}, \ldots, r_{k}$ tels que:

(1) $\left\{Z_{1}, \ldots, Z_{l}\right\}$ soit une base de $\mathfrak{g}_{\lambda}$.

(2) $B_{\lambda}\left(X_{i}, X_{j}\right)=B_{\lambda}\left(Y_{i}, Y_{j}\right)=B_{\lambda}\left(X_{i}, Y_{j}\right)-\delta_{i, j} r_{i}=0$ pour $i, j=1, \ldots, k$. Soit $X=\sum_{i=1}^{k} x_{i} X_{i}+y_{i} Y_{i}$, si l'on pose $\nu=\lambda+\lambda \circ a_{X}$, un calcul montre que pour $1 \leq i \leq k: \nu\left(X_{i}\right)=\lambda\left(X_{i}\right)+r_{i} y_{i}, \nu\left(Y_{i}\right)=\lambda\left(Y_{i}\right)-r_{i} x_{i}$ et pour $1 \leq j \leq l$, $\nu\left(Z_{j}\right)=\lambda\left(Z_{j}\right)$. On en déduit que l'on peut choisir $X$ pour que $\nu\left(X_{i}\right)=\mu\left(X_{i}\right)$ et $\nu\left(Y_{i}\right)=\mu\left(Y_{i}\right)$ pour $1 \leq i \leq k$. Comme $\nu\left(Z_{j}\right)=\mu\left(Z_{j}\right)$ pour $1 \leq j \leq l$, on a $\mu=\nu$, donce $\lambda \circ D=\lambda \circ \mathrm{ad}_{X}$.

Preuve de la proposition. Soit $V$ un supplémentaire de $\mathfrak{g}^{\prime}$ dans $\mathfrak{g}$, notons $\pi$ la projection sur $V$ correspondant à la décomposition $\mathfrak{g}=V \oplus \mathfrak{g}^{\prime}$. Alors $\mathscr{R}=\pi(\log \Gamma)$ est un réseau de $V[16$, p. 21] et l'application $\Gamma \backslash G \rightarrow \mathscr{R} \backslash V$, $x \mapsto \pi(\log x)$ est une submersion. Le but de la première partie de la preuve est de construire une submersion riemannienne à fibres totalement géodésiques. Soit $\left\{X_{1}, \ldots, X_{p}\right\}$ (resp. $\left.\left\{Y_{1}, \ldots, Y_{q}\right\}\right)$ une base de $V$ (resp. $\left.\mathfrak{g}^{\prime}\right)$, alors $\mathscr{B}=$ $\left\{X_{1}, \ldots, X_{p}, Y_{1}, \ldots, Y_{q}\right\}$ est une base de $\mathfrak{g}$ et la matrice de $\mathbf{m}_{s}$ rapport à cette base est

$$
\left(\begin{array}{cc}
\mathbf{m}_{1}(s) & \mathbf{m}_{2}(s) \\
{ }^{t} \mathbf{m}_{2}(s) & \mathbf{m}_{3}(s)
\end{array}\right)
$$

où $\mathbf{m}_{1}(s)$ (resp. $\mathbf{m}_{3}(s)$ ) est une matrice symétrique définie positive d'ordre $p$ (resp. q) et $\mathbf{m}_{2}(s)$ est une matrice à $p$ lignes et $q$ colonnes. Remarquons tout 
d'abord que tout endomorphisme $D$ de $\mathfrak{g}$ tel que $D(\mathfrak{g}) \subset \mathfrak{g}^{\prime}$ et $D\left(\mathfrak{g}^{\prime}\right)=\{0\}$ est une dérivation et que id $+D=\exp (D)$ est un élément de $\operatorname{Aut}(\mathfrak{g})$. On en déduit que pour tout $s \in I$, il existe $\varphi_{s} \in \operatorname{Aut}(G)$ tel que $\left(\varphi_{s}\right)_{*}$ ait pour matrice par rapport à $\mathscr{B}$ :

$$
\left.\begin{array}{c|c}
1 & 0 \\
\hline-\mathbf{m}_{3}^{-1}(s)^{t} \mathbf{m}_{2}(s) & 1
\end{array}\right) .
$$

Alors la matrice de $\varphi_{s}^{*} \mathbf{m}_{s}$ est

$$
\left(\begin{array}{c|c}
\mathbf{m}_{1}^{\prime}(s) & 0 \\
\hline 0 & \mathbf{m}_{3}(s)
\end{array}\right)
$$

avec $\mathbf{m}_{1}^{\prime}(s)=\mathbf{m}_{1}(s)-\mathbf{m}_{2}(s) \mathbf{m}_{3}^{-1}(s)^{t} \mathbf{m}_{2}(s)$. Or les variétés $\left(\varphi_{s}^{-1}(\Gamma) \backslash G, \varphi_{s}^{*} \mathbf{m}_{s}\right)$ et $\left(\Gamma \backslash G, \mathbf{m}_{s}\right)$ sont isométriques, donc isospectrales. Comme l'application

$$
\left(\varphi_{s}^{-1}(\Gamma) \backslash G, \varphi_{s}^{*} \mathbf{m}_{s}\right) \rightarrow\left(\mathscr{R} \backslash V, \mathbf{m}_{1}^{\prime}(s)\right), \quad x \mapsto \pi(\log x),
$$

est une submersion riemannienne à fibres totalement géodésiques, le spectre de $\left(\mathscr{R} \backslash V, \mathbf{m}_{1}^{\prime}(s)\right)$ est contenu dans celui de $\left(\varphi_{s}^{-1}(\Gamma) \backslash G, \varphi_{s}^{*} \mathbf{m}_{s}\right)$ [1, p. 188] donc, par continuité des valeurs propres, on en déduit que les tores plats $\left(\mathscr{R} \backslash V, \mathbf{m}_{1}^{\prime}(s)\right)$ sont deux à deux isospectraux, finalement $\mathbf{m}_{1}^{\prime}(s)=\mathbf{m}_{1}^{\prime}\left(s_{0}\right),\left(s_{0} \in I\right)$ et ce pour tout $s \in I$ [21, Théorème 1]. Comme, d'après le proposition précédente, pour tout $s \in I, \mathbf{m}_{\mathbf{3}}(s)=\mathbf{m}_{3}\left(s_{0}\right)$, on en déduit que $\mathbf{m}_{s}=\psi_{s}^{*} \mathbf{m}_{s_{0}}$ où $\psi_{s}=\varphi_{s_{0}}^{-1} \varphi_{s} \in$ $\operatorname{Aut}(G)$. Il ne reste plus qu'à montrer que $\psi_{s} \in \operatorname{AIA}(G, \Gamma)$.

Rappelons que $\left(\psi_{s}\right)_{*}=$ id $+D_{s}$ où $D_{s}$ est une dérivation de $\mathfrak{g}$ telle que $D_{s}(\mathfrak{g}) \subset \mathfrak{g}^{\prime}$ et $D_{s}\left(\mathfrak{g}^{\prime}\right)=\{0\}$. On va montrer que pour tout $\lambda \in \mathscr{L}^{*}, D_{s}\left(\mathfrak{g}_{\lambda}\right) \subset$ $\operatorname{Ker} \lambda \cap \mathfrak{g}_{\lambda}$, ce qui permettra de conclure.

Supposons que $\lambda\left(\mathfrak{g}^{\prime}\right)=\{0\}$, alors $\mathfrak{g}_{\lambda}=\mathfrak{g}$ et $D_{s}\left(\mathfrak{g}_{\lambda}\right) \subset \mathfrak{g}^{\prime} \subset \operatorname{Ker} \lambda$. On a donc bien $D_{s}\left(\mathfrak{g}_{\lambda}\right) \subset \operatorname{Ker} \lambda \cap \mathfrak{g}_{\lambda}$.

Supposons maintenant que $\lambda\left(\mathfrak{g}^{\prime}\right) \neq\{0\}$. Si $\mathfrak{g}_{\lambda}=\mathfrak{g}^{\prime}$, comme $D_{s}\left(\mathfrak{g}^{\prime}\right)=\{0\}$, l'inclusion à montrer est évidente. Si $\mathfrak{g}^{\prime} \neq \mathfrak{g}_{\lambda}$, comme $\mathfrak{g}^{\prime} \cap \mathscr{L}$ est un sousréseau de $\mathfrak{g}_{\lambda} \cap \mathscr{L}$, il existe une base $\left\{U_{1}, \ldots, U_{q}, V_{1}, \ldots, V_{r}\right\}$ de $\mathfrak{g}_{\lambda} \cap \mathscr{L}$ et des entiers $r_{1}, \ldots, r_{q}$ tels que $\left\{r_{1} U_{1}, \ldots, r_{q} U_{q}\right\}$ soit une base de $\mathfrak{g}_{\lambda} \cap \mathscr{L}$. Soit $\mu \in\left(\mathbb{Z}^{r}\right)^{*}=\left(\mathbb{Z} V_{1} \oplus \cdots \oplus \mathbb{Z} V_{r}\right)^{*}$, alors $\mu$ se prolonge en un élément de $\left(\mathfrak{g}_{\lambda} \cap \mathscr{L}\right)^{*}$, que l'on notera par le même lettre, en posant $\mu\left(U_{i}\right)=0$ pour $i=1, \ldots, q$. Soit donc $\mu \in\left(\mathbb{Z}^{r}\right)^{*}$, alors $\lambda$ et $\lambda+\mu$ coïncident sur $\mathfrak{g}^{\prime}$, donc $B_{\lambda+\mu}=B_{\lambda}$, $\mathfrak{g}_{\lambda}=\mathfrak{g}_{\lambda+\mu}$ et $(\lambda+\mu)\left(\mathfrak{g}_{\lambda+\mu} \cap \log \Gamma\right) \subset \mathbb{Z}$. On en déduit que pour tout $\mu \in\left(\mathbb{Z}^{r}\right)^{*}$, $\lambda+\mu$ est adapté à $\Gamma$.

Soit $s_{0} \in I$ comme avant, choisissons une base orthonormale de $\mathfrak{g}_{\lambda}$ pour $\mathbf{m}_{s_{0}}\left\{X_{1}, \ldots, X_{q}, X_{q+1}, \ldots, X_{q+r}\right\}$ telle que $\left\{X_{1}, \ldots, X_{q}\right\}$ soit une base orthonormale de $\mathfrak{g}^{\prime}$. Comme pour tout $s \in I,\left(\psi_{s}\right)_{*}\left(\mathfrak{g}_{\lambda}\right)=\mathfrak{g}_{\lambda},\left\{\left(\psi_{s}^{-1}\right)_{*}\left(X_{1}\right), \ldots\right.$, $\left.\left(\psi_{s}^{-1}\right)_{*}\left(X_{q+r}\right)\right\}$ est une base orthonormale de $\mathfrak{g}_{\lambda}$ pour $\psi_{s}^{*} \mathbf{m}_{s_{0}}=\mathbf{m}_{s}$. En utilisant la preuve de la proposition précédente, on trouve que pour tout $\mu \in\left(\mathbb{Z}^{r}\right)^{*}$ la quantité $\sum_{i=1}^{q+r}\left((\lambda+\mu)\left(\left(\psi_{s}^{-1}\right)_{*}\left(X_{i}\right)\right)\right)^{2}$ est indépendante de $s$ et en considérant le terme homogène de degré un en $\mu$ dans cette expression on trouve que pour tout $s \in I$ et pour tout $\mu \in\left(\mathbb{Z}^{r}\right)^{*}$,

$$
\sum_{i=1}^{q+r} \lambda\left(\left(\psi_{s}^{-1}\right)_{*}\left(X_{i}\right)\right) \mu\left(\left(\psi_{s}^{-1}\right)_{*}\left(X_{i}\right)\right)=\sum_{i=1}^{q+r} \lambda\left(X_{i}\right) \mu\left(X_{i}\right) .
$$

En tenant compte du fait que $\left(\psi_{s}^{-1}\right)_{*}=\mathrm{id}-D_{s}$ et que $\left(\psi_{s}^{-1}\right)_{*}\left(X_{i}\right)=X_{i}$ pour 
$1 \leq i \leq q$, on obtient

$$
\sum_{i=q+1}^{q+r} \lambda\left(X_{i}-D_{s}\left(X_{i}\right)\right) \mu\left(X_{i}-D_{s}\left(X_{i}\right)\right)=\sum_{i=q+1}^{q+r} \lambda\left(X_{i}\right) \mu\left(X_{i}\right) .
$$

Comme $\mu\left(\mathfrak{g}^{\prime}\right)=\{0\}$, on obtient finalement $\sum_{i=q+1}^{q+r} \lambda \circ D_{s}\left(X_{i}\right) \mu\left(X_{i}\right)=0$. Ceci étant vrai pour tout $\mu \in\left(\mathbb{Z}^{r}\right)^{*}$, on a forcément $\lambda \circ D_{s}\left(X_{i}\right)=0$ pour $i=$ $q+1, \ldots, q+r$. Comme $D_{s}\left(\mathfrak{g}^{\prime}\right)=\{0\}, \lambda \circ D_{s}\left(X_{i}\right)=0$ pour $i=1, \ldots, q$, on a $D_{s}\left(\mathfrak{g}_{\lambda}\right) \subset \operatorname{Ker} \lambda$. Or $D_{s}\left(\mathfrak{g}_{\lambda}\right) \subset D_{s}(\mathfrak{g}) \subset \mathfrak{g}^{\prime} \subset \mathfrak{g}_{\lambda}$, on en déduit que $D_{s}\left(\mathfrak{g}_{\lambda}\right) \subset$ $\operatorname{Ker} \lambda \cap \mathfrak{g}_{\lambda}$, donc $D_{s} \in \operatorname{AID}(\mathfrak{g}, \Gamma)$.

\section{APPENDICE A}

Le but de cet Appendice est de faire en détails les calculs utilisés dans la deuxième partie, dont on reprend les notations, pour décrire le spectre des nilvariétés de rang deux.

Dans un premier temps, on calcule le spectre $\Sigma(\lambda, \mathbf{m})$ de l'opérateur $\Delta_{\lambda, \mathbf{m}}$ défini par $\Delta_{\lambda, \mathbf{m}}=-\sum_{i=1}^{n}\left(\pi_{\lambda}\right)_{*}\left(X_{i}\right)^{2}$ où $\left\{X_{i}\right\}_{1 \leq i \leq n}$ est une base orthonormée quelconque de $\mathfrak{g}$ pour $\mathbf{m}$.

Considérons d'abord le cas où $\lambda$ est nulle sur l'algèbre dérivée $\mathfrak{g}^{\prime}$. Alors $\mathfrak{g}$ est l'unique sous-algèbre maximale subordonnée à $\lambda$ et la représentation $\pi_{\lambda}$ est le caractère définie par $\pi_{\lambda}(\exp (X))=\exp (2 \pi i \lambda(X))$ pour $x \in \mathfrak{g}$. Dans ce cas, l'espace $V_{\lambda}$ de la représentation $\pi_{\lambda}$ est de dimension 1 et l'opérateur $\Delta_{\lambda, \mathbf{m}}$ correspond à la multiplication par $4 \pi^{2} \sum_{j=1}^{n} \lambda\left(X_{j}\right)^{2}$ où $\left\{X_{j}\right\}_{1 \leq j \leq n}$ est une base orthonormée de $\mathfrak{g}$ pour la métrique $\mathbf{m}$. Donc, si $\lambda$ s'annule sur $\mathfrak{g}^{\prime}$ alors $\Sigma(\lambda, \mathbf{m})=\left\{4 \pi^{2} \sum_{j=1}^{n} \lambda\left(X_{j}\right)^{2}\right\}$

Supposons maintenant que $\lambda$ n'est pas identiquement nulle sur $\mathfrak{g}^{\prime}$. Par la suite on notera $B_{\lambda}$ la forme bilinéaire antisymétrique définie par $B_{\lambda}(X, Y)=$ $\lambda([X, Y])$ pour $X, Y \in \mathfrak{g}$ et $\mathfrak{g}_{\lambda}$ le noyau de $B_{\lambda}: \mathfrak{g}_{\lambda}=\{X \in \mathfrak{g}$ tels que $B_{\lambda}(X, Y)=0$ pour tout $Y \in \mathfrak{g}$ \} (remarquons que, par hypothèse, $\mathfrak{g}_{\lambda} \neq \mathfrak{g}$ puisque $B_{\lambda}$ n'est pas identiquement nulle). Il existe dans une base orthonormée de $\mathfrak{g}$ pour la métrique $\mathbf{m}, \mathscr{B}=\left\{U_{1}, \ldots, U_{k}, V_{1}, \ldots, V_{k}, W_{1}, \ldots, W_{l}\right\}$, et des nombres réels $0<d_{1} \leq \cdots \leq d_{k}$ tels que:

- $\left\{W_{1}, \ldots, W_{l}\right\}$ soit une base de $\mathfrak{g}_{\lambda}\left(l=\operatorname{dim} \mathfrak{g}_{\lambda}\right)$

- Pour $1 \leq i, j \leq k, 0=B_{\lambda}\left(U_{i}, U_{j}\right)=B_{\lambda}\left(V_{i}, V_{j}\right)=B_{\lambda}\left(U_{i}, V_{j}\right)-\delta_{i, j} d_{i}$. Remarquons que $\pm \overline{\mathrm{id}}_{j}$ pour $j=1, \ldots, k$ sont les valeurs propres non nulles de l'opérateur $u_{\lambda}$, antisymétrique par rapport à la métrique $\mathbf{m}$, défini par $B_{\lambda}(X, Y)=\mathbf{m}\left(X, u_{\lambda}(Y)\right)$ pour $X, Y \in \mathfrak{g}$. On a alors le résultat suivant.

1. Lemme. Avec les notations précédentes, l'espace vectoriel $\mathfrak{h}_{\lambda}=\mathfrak{g}_{\lambda} \oplus \mathbb{R} V_{1} \oplus$ $\cdots \oplus \mathbb{R} V_{k}$ est une sous-algèbre subordonnée maximale par rapport à $\lambda$.

Preuve. $\mathfrak{h}_{\lambda}$ est une sous-algèbre. En effet, comme $\mathfrak{g}$ est nilpotente de rang 2 , l'algèbre dérivée $\mathfrak{g}^{\prime}$ est contenue dans le centre, donc dans $\mathfrak{g}_{\lambda}$. Puisque $\mathfrak{h}_{\lambda}$ contient $\mathfrak{g}_{\lambda}, \mathfrak{h}_{\lambda}$ contient $\mathfrak{g}^{\prime}$, donc $\mathfrak{h}_{\lambda}$ est un idéal et, a fortiori, une sous-algèbre.

$\mathfrak{h}_{\lambda}$ est subordonée maximale. En effet, la restriction de $B_{\lambda}$ à $\mathfrak{h}_{\lambda}$ est, par construction de $\mathfrak{h}_{\lambda}$, nulle et il est clair que $\mathfrak{h}_{\lambda}$ est un sous-espace vectoriel totalement isotrope de dimension maximale, donc, a fortiori, $\mathfrak{h}_{\lambda}$ est une sousalgèbre subordonnée maximale par rapport à $\lambda$.

Posons $H_{\lambda}=\exp \left(\mathfrak{h}_{\lambda}\right)$ et définissons le caractère $\bar{\lambda}$ sur $H_{\lambda} \operatorname{par} \bar{\lambda}(\exp X)=$ $\exp (2 \pi i \lambda(X))$ où $X \in \mathfrak{h}_{\lambda}$. On va obtenir une expression de la représentation 
$\pi_{\lambda}$ induite par le couple $\left(\bar{\lambda}, H_{\lambda}\right)$. Remarquons que tout sous-espace vectoriel de $\mathfrak{g}$ qui contient $\mathfrak{g}^{\prime}$ est un idéal, donc une sous-algèbre. On en déduit que les espaces $\mathfrak{h}_{i}=\mathfrak{h}_{\lambda} \oplus \mathbb{R} U_{1} \oplus \cdots \oplus \mathbb{R} U_{i} \quad(1 \leq i \leq k)$ sont des sous-algèbres, donc, d'après la Proposition I.1, l'application

$$
\begin{aligned}
\mathbb{R}^{k} \times H_{\lambda} & \rightarrow G \\
(t, h) & \mapsto h \prod_{j=1}^{k} \exp \left(t_{j} X_{j}\right)
\end{aligned}
$$

est un difféomorphisme. Reprenons les notations de la première partie:

$$
\begin{aligned}
& \mathscr{H}_{0}=\left\{f \in C^{0}(G) \text { telles que pour } x \in G \text { et } h \in H_{\lambda}, f(h x)=\bar{\lambda}(h) f(x)\right\}, \\
& \mathscr{H}_{1}=\left\{f \in \mathscr{H}_{0} \text { telles que } \int_{\mathbb{R}^{k}}\left|f\left(\prod_{j=1}^{k} \exp \left(t_{j} X_{j}\right)\right)\right|^{2} d t<+\infty\right\} .
\end{aligned}
$$

Soit, enfin, $\mathscr{H}$ le complété de $\mathscr{H}_{1}$ pour la norme $L^{2} ; \mathscr{H}$ s'identifie naturellement à $L_{\mathbb{C}}^{2}\left(\mathbb{R}^{k}\right)$ par l'isométrie $\alpha: \mathscr{H} \rightarrow L_{\mathbb{C}}^{2}\left(\mathbb{R}^{k}\right)$ définie par $\alpha(f)(t)=$ $f\left(\prod_{j=1}^{k} \exp \left(t_{j} X_{j}\right)\right)$ où $f \in \mathscr{H}$ et $t \in \mathbb{R}^{k}$.

La représentation induite $\pi_{\lambda}=\pi\left(\lambda, H_{\lambda}\right)$ est définie par: $\left(\pi_{\lambda}(x) f\right)(y)=$ $f(y x)$ où $x, y \in G$ et $f \in \mathscr{H}$. Notons $\pi_{\lambda}^{\prime}$ la représentation définie par $\pi_{\lambda}^{\prime}(x)=\alpha \circ \pi_{\lambda}(x) \circ \alpha^{-1}$ où $x \in G$. Alors $\pi_{\lambda}^{\prime}$ est unitairement équivalente à $\pi_{\lambda}$ et son espace de représentation est $L_{\mathbb{C}}^{2}\left(\mathbb{R}^{k}\right)$. On va obtenir une expression "simple" de $\pi_{\lambda}^{\prime}$.

Soit $x=h_{0} \prod_{j=1}^{k} \exp \left(s_{j} U_{j}\right)$ où $h_{0} \in H_{\lambda}$ et $s=\left(s_{1}, \ldots, s_{k}\right) \in \mathbb{R}^{k}$, on va calculer $\pi_{\lambda}^{\prime}(x)$. Soit $f \in L_{\mathbb{C}}^{2}\left(\mathbb{R}^{k}\right)$ :

$$
\left(\pi_{\lambda}^{\prime}(x) f\right)(t)=\left(\alpha^{-1}(f)\right)\left(\prod_{j=1}^{k} \exp \left(t_{j} U_{j}\right) h_{0} \prod_{j=1}^{k} \exp \left(s_{j} U_{j}\right)\right) .
$$

Or

$$
\begin{aligned}
& \prod_{j=1}^{k} \exp \left(t_{j} U_{j}\right) h_{0}=\left[\prod_{j=1}^{k} \exp \left(t_{j} U_{j}\right), h_{0}\right] h_{0} \prod_{j=1}^{k} \exp \left(t_{j} U_{j}\right), \\
& \prod_{j=1}^{k} \exp \left(t_{j} U_{j}\right) \prod_{j=1}^{l} \exp \left(s_{j} U_{j}\right) \\
& \quad=\prod_{j=1}^{l} \exp \left(\left(t_{j}+s_{j}\right) U_{j}\right) \exp \left(-\sum_{1 \leq j<l \leq k} t_{l} s_{j}\left[U_{j}, U_{l}\right]\right) .
\end{aligned}
$$

On en déduit que

$$
\left(\pi_{\lambda}^{\prime}(x) f\right)(t)=\bar{\lambda}\left(\left[\prod_{j=1}^{k} \exp \left(t_{j} U_{j}\right), h_{0}\right] h_{0} \exp \left(-\sum_{1, \ldots, j<l, \ldots, k} s_{j} t_{l}\left[U_{j}, U_{l}\right]\right)\right) b(t+s) .
$$


Finalement, en tenant compte du fait que

$$
\left[\exp \left(\sum_{j=1}^{k} t_{j} U_{j}\right), h_{0}\right]=\exp \left(\left[\sum_{j=1}^{k} t_{j} U_{j}, \log h_{0}\right]\right)
$$

(puisque $G$ est nilpotent de rang deux) et que la restriction de $B_{\lambda}$ à $\mathbb{R} U_{1} \oplus$ $\cdots \oplus \mathbb{R} U_{k}$ est nulle, on obtient

$$
\left(\pi_{\lambda}^{\prime}(x) f\right)(t)=f(t+s) \exp \left(2 \pi i \lambda\left(\log h_{0}+\left[\sum_{j=1}^{k} t_{j} U_{j}, \log h_{0}\right]\right)\right)
$$

où $t \in \mathbb{R}^{k}$ et $x=h_{0} \prod_{j=1}^{k} \exp \left(s_{j} U_{j}\right)$ avec $h_{0} \in H_{\lambda}$ et $s=\left(s_{1}, \ldots, s_{k}\right) \in \mathbb{R}^{k}$.

Comme $\pi_{\lambda}$ et $\pi_{\lambda}^{\prime}$ sont unitairement équivalentes, il suffit de calculer les valeurs propres de l'opérateur $\Delta_{\lambda, \mathrm{m}}^{\prime}$ défini par $\Delta_{\lambda, \mathrm{m}}^{\prime}=-\sum_{i=1}^{n}\left(\pi_{\lambda}^{\prime}\right)_{*}\left(X_{i}\right)^{2}$ où $\left\{X_{i}\right\}_{1 \leq i \leq n}$ est une base orthonormée quelconque de $\mathfrak{g}$ pour la métrique $\mathbf{m}$. Choisissons comme base orthonormée le base $\mathscr{B}$ considérée plus haut. Soit $f \in C^{\infty}\left(\mathbb{R}^{k}\right)$.

$$
\left(\pi_{\lambda}^{\prime}\left(\exp \left(s U_{j}\right)\right) f\right)(t)=f(t+\underbrace{(0, \ldots, 0, s, 0, \ldots, 0)}_{i^{e} \text { place }}) .
$$

On en déduit que $\left(\left(\pi_{\lambda}^{\prime}\right)_{*}\left(U_{j}\right) f\right)(t)=\partial f(t) / \partial t_{i}$.

$$
\begin{aligned}
\left(\pi_{\lambda}^{\prime}\left(\exp \left(s V_{j}\right)\right) f\right)(t) & =f(t) \exp \left[2 \pi i \lambda\left(s V_{j}+s\left[\sum_{l=1}^{k} t_{l} U_{l}, V_{j}\right]\right)\right] \\
& =f(t) \exp \left[2 \pi i s\left(\lambda\left(V_{j}\right)+d_{j} t_{j}\right)\right] .
\end{aligned}
$$

On en déduit que $\left(\left(\pi_{\lambda}^{\prime}\right)_{*}\left(V_{j}\right) f\right)(t)=f(t) 2 \pi i\left(\lambda\left(V_{j}\right)+d_{j} t_{j}\right)$.

$$
\left(\pi_{\lambda}^{\prime}\left(\exp \left(s W_{j}\right)\right) f\right)(t)=f(t) \exp \left[2 \pi i \lambda\left(s W_{j}\right)\right] .
$$

On en déduit que $\left(\left(\pi_{\lambda}^{\prime}\right)_{*}\left(W_{j}\right) f\right)(t)=2 \pi i \lambda\left(W_{j}\right) f(t)$.

On a donc l'expresion suivante:

$$
\Delta_{\lambda, \mathbf{m}}^{\prime} f(t)=-\sum_{i=1}^{k} \frac{\partial^{2} f}{\partial t_{i}^{2}}(t)+4 \pi^{2}\left(\sum_{i=1}^{k}\left(\lambda\left(V_{i}\right)+d_{i} t_{i}\right)^{2}+\sum_{j=1}^{l} \lambda\left(W_{j}\right)^{2}\right) f(t) .
$$

Remarquons que $\Delta_{\lambda, \mathbf{m}}^{\prime}$ est unitairement conjugué à l'opérateur $\Delta_{\lambda, \mathrm{m}}^{\prime \prime}$ défini par

$$
\Delta_{\lambda, \mathbf{m}}^{\prime \prime} f(t)=-\sum_{i=1}^{k} \frac{\partial^{2} f}{\partial t_{i}^{2}}(t)+4 \pi^{2}\left(\sum_{i=1}^{k} d_{i}^{2} t_{i}^{2}+\sum_{j=1}^{l} \lambda\left(W_{j}\right)^{2}\right) f(t) .
$$

En effet, si $a$ est le vecteur de $\mathbb{R}^{k}$ dont la $j$-ème composante est $\lambda\left(V_{j}\right) / d_{j}$ et $T$ l'opérateur unitaire de $L_{\mathbb{C}}^{2}\left(\mathbb{R}^{k}\right)$ défini par $(T f)(t)=f(t-a) \quad\left(f \in L_{\mathbb{C}}^{2}\left(\mathbb{R}^{k}\right)\right.$ et $\left.t \in \mathbb{R}^{k}\right)$, alors on a la relation suivante: $T \circ \Delta_{\lambda, \mathbf{m}}^{\prime}=\Delta_{\lambda, \mathrm{m}}^{\prime \prime} \circ T$.

D'après ce que l'on vient de voir, il suffit de calculer les valeurs propres de l'opérateur $\Delta_{\lambda, \mathrm{m}}^{\prime \prime}$. Pour cela, on va utiliser les fonctions d'Hermite définies par

$$
H_{p}(t)=\exp \left(\frac{\|t\|^{2}}{2}\right) \times \frac{\partial^{|p|}}{\partial t^{p}} \exp \left(-\|t\|^{2}\right)
$$


où $\|\cdot\|$ désigne la norme euclidienne usuelle sur $\mathbb{R}^{k}$ et $p=\left(p_{1}, \ldots, p_{k}\right)$ est un élément de $\mathbb{N}^{k}$ (on a posé $|p|=\sum_{i=1}^{k} p_{i}$ ). Il est connu [9] que $\left\{H_{p}\right\}_{p \in \mathbb{N}^{k}}$ est une base orthogonale de $L_{\mathbb{C}}^{2}\left(\mathbb{R}^{k}\right)$ et que pour tout $p=\left(p_{1}, \ldots, p_{k}\right)$ dans $\mathbb{N}^{k}$, on a la relation suivante:

$$
t_{i}^{2} H_{p}(t)-\frac{\partial^{2} H_{p}}{\partial t_{i}^{2}}(t)=\left(2 p_{i}+1\right) H_{p}(t) \quad \text { pour } i=1, \ldots, k
$$

Donc, si l'on pose $K_{p}(t)=H_{p}\left(t_{1}\left(2 \pi d_{1}\right)^{1 / 2}, \ldots, t_{k}\left(2 \pi d_{k}\right)^{1 / 2}\right)$, on a la relation suivante:

$$
-\frac{\partial^{2} K_{p}}{\partial t_{i}^{2}}(t)=\left(-4 \pi^{2} d_{i}^{2} t_{i}^{2}+2 \pi\left(2 p_{i}+1\right) d_{i}\right) K_{p}(t) \quad \text { pour } i=1, \ldots, k .
$$

Un calcul facile montre alors que: $\Delta_{\lambda, \mathbf{m}}^{\prime \prime} K_{p}=\mu(\lambda, p, \mathbf{m}) K_{p}$ où l'on a posé:

$$
\mu(\lambda, p, \mathbf{m})=4 \pi^{2} \sum_{j=1}^{l}\left(\lambda\left(W_{j}\right)\right)^{2}+2 \pi \sum_{i=1}^{k}\left(2 p_{i}+1\right) d_{i} .
$$

On déduit donc que si $\lambda$ n'est pas identiquement nulle sur $\mathfrak{g}^{\prime}, \Sigma(\lambda, \mathbf{m})=$ $\left\{\mu(\lambda, p, \mathbf{m}) ; p \in \mathbb{N}^{k}\right\}$ où $\mu(\lambda, p, \mathbf{m})$ est le nombre défini ci-dessus et où, rappelons-le:

(1) $\left\{W_{j}\right\}_{1 \leq j \leq l}$ est une base orthonormée de $\mathfrak{g}_{\lambda}$ pour la métrique $\mathbf{m}$.

(2) $\pm \mathrm{id}_{j}$ pour $j=1, \ldots, k$ sont les valeurs propres non nulles de l'opérateur $u_{\lambda}$, antisymétrique par rapport à $\mathbf{m}$, défini par

$$
B_{\lambda}(X, Y)=\mathbf{m}\left(X, u_{\lambda}(Y)\right) \text { pour } X, Y \in \mathfrak{g} .
$$

2. Remarque. D’après les résultats de la première partie, les nombres $\mu(\lambda, p, \mathbf{m})$ ne doivent dépendre que de l'orbite de $\lambda$. Or si $\lambda^{\prime}$ est dans $\mathscr{O}(\lambda)$, il existe $X$ dans $\mathfrak{g}$ tel que $\lambda^{\prime}=\lambda+\lambda \circ \operatorname{ad}_{X}$ (puisque $\left.\left(I_{\exp X}\right)_{*}=\mathrm{Id}+\operatorname{ad}_{X}\right)$. Donc si l'on définit $B_{\lambda^{\prime}}(X, Y)=\lambda^{\prime}([X, Y])$ pour $X, Y$ dans $\mathfrak{g}, B_{\lambda^{\prime}}=B_{\lambda}$ puisque $\mathfrak{g}$ est nilpotente de rang deux. On en déduit que $\mathfrak{g}_{\lambda}=\mathfrak{g}_{\lambda^{\prime}}$ et que, avec des notations évidentes, $d_{j}^{\prime}=d_{j}$ pour $j=1, \ldots, k \quad\left(k=\left(\operatorname{dim} \mathfrak{g}-\operatorname{dim} \mathfrak{g}_{\lambda}\right) / 2\right)$. D'autre part, si $Y \in \mathfrak{g}_{\lambda}, \lambda^{\prime}(Y)=\lambda(Y)+\lambda([X, Y])=\lambda(Y)$, ce qui montre bien que $\mu(\lambda, p, \mathbf{m})=\mu\left(\lambda^{\prime}, p, \mathbf{m}\right)$.

La fin de cette partie est consacrée à l'étude de l'ensemble $\mathscr{A}(\Gamma)$, c'est-à-dire à la caractérisation des $\lambda$ dans $\mathfrak{g}^{*}$ tels que la représentation $\pi_{\lambda}$ apparaisse dans la représentation quasi-régulière de $G$ dans $L_{\mathbb{C}}^{2}(\Gamma \backslash G)$, et des nombres $m_{\lambda}$ pour $\lambda$ dans $\mathscr{A}(\Gamma)$.

Rappelons que si $\Gamma$ et un sous-groupe uniforme de $G, \log \Gamma$ engendre $\mathfrak{g}$ [18]. Soit $\mathscr{L}$ le réseau engendré par $\log \Gamma(\log \Gamma$ n'est pas supposé être un réseau) et $\mathfrak{g}_{\mathbb{Q}}$ l'ensemble des combinaisons linéaiures à coefficients dans $\mathbb{Q}$ d'éléments de $\mathscr{L} \quad\left(\mathfrak{g}_{\mathbb{Q}} \simeq \mathscr{L} \otimes_{\mathbb{Z}} \mathbb{Q}\right)$, alors les constantes de structure de $\mathfrak{g}$ par rapport à n'importe quelle base formée d'éléments de $\mathfrak{g}_{\mathbb{Q}}$ sont rationnelles et $\mathfrak{g}_{\mathbb{Q}}$ peut donc être muni d'une structure d'algèbre de Lie rationnelle [18]. Par la suite, on dira qu'une sous-algèbre $\mathfrak{h}$ de $\mathfrak{g}$ est rationnelle (pour la structure rationnelle induite par $\Gamma$ ) si $\operatorname{dim}_{\mathbb{R}} \mathfrak{h}=\operatorname{dim}_{\mathbb{Q}} \mathfrak{h} \cap \mathfrak{g}_{\mathbb{Q}}$. D'autre part, si $\lambda$ est dans $\mathfrak{g}^{*}$, on appelle polarisation de $\lambda$ une sous-algèbre subordonnée maximale par rapport à $\lambda$ telle que $\operatorname{dim} \mathfrak{h}=\left(\operatorname{dim} \mathfrak{g}+\operatorname{dim} \mathfrak{g}_{\lambda}\right) / 2\left(\mathfrak{g}_{\lambda}\right.$ désigne toujours le noyau de la forme bilinéaire $B_{\lambda}$ définie par $B_{\lambda}(X, Y)=\lambda([X, Y])$ pour $X, Y$ dans $\mathfrak{g})$. 
Par la suite, on va considérer les couples $(\bar{\lambda}, H)$ où $\lambda$ est un élément de $\mathfrak{g}^{*}, \mathfrak{h}$ une polarisation de $\lambda$ et $\bar{\lambda}$ le caractère défini sur $H=\exp (\mathfrak{h})$ par $\bar{\lambda}(\exp X)=\exp (2 \pi i \lambda(X))$ pour $X \in \mathfrak{h}$. Un tel couple $(\bar{\lambda}, H)$ est appelé un point entier si $\mathfrak{h}$ est une polarisation rationnelle et si $\bar{\lambda}(H \cap \Gamma)=1$.

Remarquons que $G$ opère sur les couples $(\bar{\lambda}, H)$ de la manière suivante: $x \cdot(\bar{\lambda}, H)=\left(\bar{\lambda} \circ I_{x}, I_{x^{-1}}(H)\right) \quad\left(I_{x}\right.$ désigne toujours l'automorphisme intérieur $y \mapsto x y x^{-1}$ pour $\left.y \in G\right)$. On vérifie facilement que le stabilisateur de $(\bar{\lambda}, H)$ pour cette action est $H$ et que $\Gamma$ opère sur l'ensemble des points entiers. Finalement, d'après les résultats de la première partie, un couple $(\bar{\lambda}, H)$ induit une représentation unitaire irréductible. Ceci étant dit, on a le résultat suivant [13].

3. Proposition. $S i(\bar{\lambda}, H)$ induit la représentation $\pi$, alors $\pi$ apparaît dans la représentation quasi-régulière de $G$ dans $L_{\mathbb{C}}^{2}(\Gamma \backslash G)$ avec une multiplicité égale au nombre de $\Gamma$-orbites dans l'ensembie des points entiers de la $G$-orbite de $(\bar{\lambda}, H)$ (étant entendu que $\pi$ apparaît si et seulement si il existe un point entier dans la $G$-orbite de $(\bar{\lambda}, H)$.

Cette proposition est vraie pour tout groupe $G$ nilpotent simplement connexe possédant un sous-groupe uniforme. On va voir que, dans le cas où $G$ est de rang 2, cette proposition prend une forme beaucoup plus explicite. Par la suite, on supposera donc toujours que $G$ est un groupe de Lie nilpotent simplement connexe de rang deux.

On va avoir besoin de construire une base de $\mathfrak{g}$ qui soit adaptée au problème.

4. Lemme. Soit $\lambda \in \mathfrak{g}^{*}$ dont la restriction à $\mathfrak{g}^{\prime}$ est non nulle et tel que $B_{\lambda}(X, Y)$ $\in \mathbb{Z}$ pour $X, Y \in \log \Gamma$, alors il existe une base de $\mathfrak{g},\left\{U_{1}, \ldots, U_{k}, V_{1}, \ldots, V_{k}\right.$, $\left.W_{1}, \ldots, W_{l}\right\} \quad(2 k+l=\operatorname{dim} \mathfrak{g})$ formée d'éléments de $\log \Gamma$ et des entiers $r_{1}, \ldots, r_{k}$ tels que

(1) $B_{\lambda}\left(U_{i}, U_{j}\right)=B_{\lambda}\left(V_{i}, V_{j}\right)=B_{\lambda}\left(U_{i}, V_{j}\right)-r_{i} \delta_{i, j}=0$ pour $1 \leq i \leq j \leq k$.

(2) $\left\{W_{1}, \ldots, W_{l}\right\}$ est une base de $\mathfrak{g}_{\lambda}\left(l=\operatorname{dim} \mathfrak{g}_{\lambda}\right)$.

(3) $\left\{W_{1}, \ldots, W_{q}\right\}$ est une base de $\mathfrak{g}^{\prime}\left(q=\operatorname{dim} \mathfrak{g}^{\prime}\right)$.

(4) $\mathfrak{g}^{\prime} \cap \log \Gamma=\bigoplus_{i=1}^{q} \mathbb{Z} W_{i}$.

Preuve. Notons $\mathscr{L}$ le réseau engendré par $\log \Gamma$. Soit $\mathscr{I}_{1}=\left\{B_{\lambda}(X, Y)\right.$; $X, Y \in \mathscr{L}\} \cap \mathbb{R}^{+*}$ et $r_{1}=\inf \mathscr{I}_{1}$. Remarquons que $r_{1}$ est non nul puisque $\lambda$ est non nulle sur $\mathfrak{g}^{\prime}$ et que $\mathscr{L}$ engendre $\mathfrak{g}$. Il existe donc $U_{1}^{0}$ et $V_{1}^{0}$ dans $\mathscr{L}$ tels que $B_{\lambda}\left(U_{1}^{0}, V_{1}^{0}\right)=r_{1}$. Alors $\mathfrak{g}=\left(\mathbb{R} U_{1}^{0} \oplus \mathbb{R} V_{1}^{0}\right) \oplus \mathscr{B}, \mathscr{B}$ étant l'orthogonal de $\mathbb{R} U_{1}^{0} \oplus \mathbb{R} V_{1}^{0}$ pour $B_{\lambda}$. On va voir que $\mathscr{L}=\mathbb{Z} U_{1}^{0} \oplus \mathbb{Z} V_{1}^{0} \oplus(\mathscr{B} \cap \mathscr{L})$. En effet, soit $X$ dans $\mathscr{L}, X=\alpha U_{1}^{0}+\beta V_{1}^{0}+X^{\prime}$ où $X^{\prime} \in \mathscr{B}$. Ecrivons $\alpha=[\alpha]+\alpha^{\prime}$ où $[\alpha]$ désigne la partie entière de $\alpha$ et $\alpha^{\prime} \in[0,1[$, alors $X-[\alpha] U_{1}^{0} \in \mathscr{L}$ et $B_{\lambda}\left(X-[\alpha] U_{1}^{0}, V_{1}^{0}\right)=\alpha^{\prime} r_{1}$. Comme $\alpha^{\prime} \in[0,1[$, on a forcément $\alpha^{\prime}=0$. On en déduit que $\alpha \in \mathbb{Z}$, puis, par un raisonnement analogue, on montre que $\beta \in \mathbb{Z}$, ce qui montre bien que $\mathscr{L}=\mathbb{Z} U_{1}^{0} \oplus \mathbb{Z} V_{1}^{0} \oplus$ $(\mathscr{B} \cap \mathscr{L})$. En considérant $\mathscr{I}_{2}=\left\{B_{\lambda}(X, Y) ; X, Y \in(\mathscr{B} \cap \mathscr{L})\right\} \cap \mathbb{R}^{+*}$ et $r_{2}=\inf \mathscr{I}_{2}$, on peut continuer le raisonnement et construire une base de $\mathscr{L}$, $\left\{U_{1}^{0}, \ldots, U_{k}^{0}, V_{1}^{0}, \ldots, V_{k}^{0}, W_{1}^{0}, \ldots, W_{l}^{0}\right\}$ qui vérifie les propriétés (1) et (2) du lemme.

Remarquons que $\log \Gamma \cap \mathfrak{g}^{\prime}$ est un sous-réseau de $\mathfrak{g}_{\lambda} \cap \mathscr{L}$, il existe donc une base $\left\{W_{1}^{1}, \ldots, W_{l}^{1}\right\}$ de $\mathfrak{g}_{\lambda} \cap \mathscr{L}$ et des entiers $s_{1}, \ldots, s_{q}\left(q=\operatorname{dim} \mathfrak{g}^{\prime}\right)$ tels que 
$\left\{s_{1} W_{1}^{1}, \ldots, s_{q} W_{q}^{1}\right\}$ soit une base de $\log \Gamma \cap \mathfrak{g}^{\prime}$. Donc si l'on pose $W_{i}^{2}=s_{i} W_{i}^{1}$ pour $1 \leq i \leq q$ et $W_{i}^{2}=W_{i}^{1}$ pour $q<i \leq l,\left\{U_{1}^{0}, \ldots, U_{k}^{0}, V_{1}^{0}, \ldots, V_{k}^{0}\right.$, $\left.W_{1}^{2}, \ldots, W_{l}^{2}\right\}$ est une base de $\mathfrak{g}$ vérifiant les propriétés (1), (2), (3), (4) du lemme. Il ne reste plus qu'à montrer que l'on peut choisir une base de $\mathfrak{g}$ vérifiant les quatre propriétés précédentes et constituée d'éléments de $\log \Gamma$. Or si $X \in \mathscr{L}, X=\sum_{i=1}^{N} p_{i} X_{i}$ où $p_{i} \in \mathbb{Z}$ et $X_{i} \in \log \Gamma$ pour $1 \leq i \leq N$, donc

$$
\exp (X)=\prod_{i=1}^{N} \exp \left(p_{i} X_{i}\right) \exp \left(-\frac{1}{2} \sum_{i<j} p_{i} p_{j}\left[X_{i}, X_{j}\right]\right) .
$$

On en déduit que $X+\frac{1}{2} \sum_{i<j} p_{i} p_{j}\left[X_{i}, X_{j}\right] \in \log \Gamma$. On peut donc choisir des éléments $U_{i}^{3}$ (resp. $V_{i}^{3}, W_{j}^{3}$ ) de $\mathfrak{g}^{\prime} \cap \mathscr{L}$ tels que $U_{i}=U_{i}^{0}+U_{i}^{3}$ (resp. $V_{i}=$ $\left.V_{i}^{0}+V_{i}^{3} ; W_{j}=W_{j}^{2}+W_{j}^{3}\right)$ soit un élément de $\log \Gamma$ pour $1 \leq i \leq k$ et $1 \leq j \leq l$ (on choisit évidemment $W_{i}^{3}=0$ pour $1 \leq i \leq q$ ). Comme $\mathfrak{g}^{\prime} \subset \mathfrak{g}_{\lambda}$, la base $\left\{U_{1}, \ldots, U_{k}, V_{1}, \ldots, V_{k}, W_{1}, \ldots, W_{l}\right\}$ est une base de $\mathfrak{g}$ formée d'éléments de $\log \Gamma$ vérifiant les propriétés (1), (2), (3), (4).

Avant de continuer, on a besoin de résultats sur la structure des groupes de Lie nilpotents simplement connexe et de leur sous-groupe uniforme.

5. Définition. Soient $\Gamma$ un sous-groupe uniforme d'un groupe de Lie nilpotent simplement connexe de dimension $n$ et $\gamma_{1}, \ldots, \gamma_{n}$ des éléments de $\Gamma$. On dit que $\left\{\gamma_{1}, \ldots, \gamma_{n}\right\}$ est une base canonique de $\Gamma$ si $\mathbb{Z}^{n}$

(a) Tout élément $\gamma$ de $\Gamma$ s'écrit sous la forme $\gamma=\prod_{i=1}^{n} \gamma_{i}^{p_{i}}$ où $\left(p_{1}, \ldots, p_{n}\right) \in$

(b) L'ensemble $\Gamma_{i}=\left\{\gamma_{i}^{p_{i}} \cdots \gamma_{j}^{p_{j}} \cdot \gamma_{n}^{p_{n}}\right.$ où $p_{j} \in \mathbb{Z}$ pour $\left.i \leq j \leq n\right\}$ est un sous-groupe normal de $\Gamma$.

(c) Le groupe $\Gamma_{i} / \Gamma_{i+1}$ est isomorphe à $\mathbb{Z}$ pour $1 \leq i \leq n-1$.

6. Remarque. On vérifie facilement, en utilisant les conditions (b) et (c), que l'écriture $\gamma=\prod_{i=1}^{n} \gamma_{i}^{p_{i}}$ est unique.

On va voir maintenant que la base de $\mathfrak{g}$ construite au lemme A.4 permet de construire une base canonique de $\Gamma$. De manière plus précise, on a le résultat suivant:

7. Lemme. Soient $G$ un groupe de Lie nilpotent simplement connexe de rang $2, \Gamma$ un sous-groupe uniforme de $G$ et $\lambda$ un élément de $\mathfrak{g}^{*}$ tel que $B_{\lambda}(X, Y) \in$ $\mathbb{Z}$ pour $X, Y \in \log \Gamma$, soit $\left\{X_{i}\right\}_{1 \leq i \leq n}(n=\operatorname{dim} G)$ une base de $\mathfrak{g}$ formée d'éléments de $\log \Gamma$ vérifiant les propriétés (1), (2), (3), (4) du lemme A.4, alors $\left\{\exp \left(X_{1}\right), \ldots, \exp \left(X_{n}\right)\right\}$ est une base canonique de $\Gamma$.

Preuve. On peut écrire cette base sous la forme $\left\{Y_{1}, \ldots, Y_{s}, Z_{1}, \ldots, Z_{q}\right\} \quad(q=$ $\left.\operatorname{dim} \mathfrak{g}^{\prime} ; s=\operatorname{dim} \mathfrak{g}-l\right)$ où $\left\{Z_{1}, \ldots, Z_{q}\right\}$ est une base $\operatorname{de} \log \Gamma \cap \mathfrak{g}^{\prime}$. Soit $p$ la projection de $G$ sur $G / G^{\prime}$, alors $p(\Gamma)$ est un sous-groupe uniforme de $G / G^{\prime}$. En effet:

( $\alpha) p(\Gamma)$ est une sous-groupe discret de $G / G^{\prime}$. En effet, supposons qu'il existe une suite $\left\{\gamma_{n}\right\}_{n \geq 0}$ d'éléments de $\Gamma$ telle que la suite $\left\{p\left(\gamma_{n}\right)\right\}_{n \geq 0}$ converge vers $p(1)$ ( 1 est l'élément neutre de $G$ ) et que ses termes soient deux à deux distincts. Il existe une suite $\left\{x_{n}\right\}_{n \geq 0}$ d'éléments de $G^{\prime}$ telle que $\lim _{n \rightarrow+\infty} \gamma_{n} x_{n}=$ 1. Or $G^{\prime} \cap \Gamma$ est un sous-groupe uniforme de $G^{\prime}$ [18], on peut donc écrire 
$x_{n}=\delta_{n} y_{n}$ où $\delta_{n} \in \Gamma \cap G^{\prime}$ et $\left\{y_{n}\right\}_{n \geq 0}$ est une suite d'éléments de $G^{\prime}$ admettant une sous-suite $\left\{y_{a_{n}}\right\}_{n \geq 0}$ convergente. Comme $\lim _{n \rightarrow+\infty} \gamma_{a_{n}} \delta_{a_{n}} y_{a_{n}}=1$, la suite $\left\{\gamma_{a_{n}} \delta_{a_{n}}\right\}_{n \geq 0}$ est convergente, donc constante à partir d'un certain rang, ce qui contredit le fait que les termes de la suite $\left\{p\left(\gamma_{n}\right)\right\}_{n \geq 0}$ sont deux à deux distincts.

( $\beta) p(\Gamma) \backslash G / G^{\prime}$ est compact. En effet la projection $p: G \rightarrow G / G^{\prime}$ induit une projection continue $p^{\prime}: \Gamma \backslash G \rightarrow p(\Gamma) \backslash G / G^{\prime}$. Ce qui montre bien que $p(\Gamma)$ est un sous-groupe uniforme de $G / G^{\prime}$.

Comme $G / G^{\prime}$ est un groupe abélien simplement connexe, $p(\Gamma)$ est un réseau et, d'après la preuve du lemme A.4, il est clair que $\left\{p\left(\exp \left(Y_{1}\right)\right), \ldots, p\left(\exp \left(Y_{s}\right)\right)\right\}$ est une base de $p(\Gamma)$. Comme $\left\{Z_{1}, \ldots, Z_{l}\right\}$ est une base de $\log \Gamma \cap \mathfrak{g}^{\prime}$, tout élément $\gamma$ de $\Gamma$ s'écrit sous la forme

$$
\gamma=\prod_{i=1}^{s} \exp \left(a_{i} Y_{i}\right) \prod_{j=1}^{l} \exp \left(b_{j} Y_{j}\right) \quad \text { où } a_{i}, b_{j} \in \mathbb{Z}(1 \leq i \leq s ; 1 \leq j \leq l) .
$$

Les propriétés (b) et (c) proviennent directement du fait que $G^{\prime}$ est abélien et que $\left\{p\left(\exp \left(Y_{1}\right)\right), \ldots, p\left(\exp \left(Y_{s}\right)\right)\right\}$ est une base du réseau $p(\Gamma)$.

Le théorème suivant, dû à Mal'cev [12], permet d'obtenir un système de coordonnées sur $G$ qui est adapté au problème qui nous intéresse.

8. Théorème. Soient $G$ un groupe de Lie nilpotent simplement connexe de dimension $n$ et $\Gamma$ un sous-groupe uniforme de $G$. Si $\left\{\gamma_{1}, \ldots, \gamma_{n}\right\}$ est une base canonique de $\Gamma$ et $X_{i}=\log \gamma_{i}$ pour $1 \leq i \leq n$, alors

(a) L'application $\mathbb{R}^{k} \rightarrow G, t=\left(t_{1}, \ldots, t_{n}\right) \mapsto \prod_{i=1}^{n} \exp \left(t_{i} X_{i}\right)$ est un difféomorphisme.

(b) Pour $1 \leq i \leq n, G_{i}=\left\{\prod_{j=i}^{n} \exp \left(t_{j} X_{j}\right) ;\left(t_{i}, \ldots, t_{n}\right) \in \mathbb{R}^{n-i+1}\right\}$ est un sous-groupe fermé normal dans $G$.

(c) Pour $1, \ldots, i \leq n-1$, le groupe $G_{i} / G_{i+1}$ est isomorphe à $\mathbb{R}$.

On va maintenant obtenir une version plus explicite de la proposition A.3.

Avant d'énoncer les résultats obtenus, remarquons que $B_{\lambda}$ induit une forme bilinéaire antisymétrique non dégénérée sur $\mathfrak{g} / \mathfrak{g}_{\lambda}$ que l'on notera $\widetilde{B}_{\lambda}$ et que, bien que $\log \Gamma$ ne soit pas, a priori, un réseau, son image $\mathscr{L}_{\lambda}$ dans $\mathfrak{g} / \mathfrak{g}_{\lambda}$ en est un (puisque si $X, Y \in \log \Gamma$, alors $X+Y+\frac{1}{2}[X, Y] \in \log \Gamma$ et $[X, Y] \in \mathfrak{g}^{\prime} \subset \mathfrak{g}_{\lambda}$ ).

9. Proposition. Soient $G$ un groupe de Lie nilpotent simplement connexe de rang deux, $\Gamma$ un sous-groupe uniforme de Get $\lambda$ un élément de $\mathfrak{g}^{*}$. Alors, avec les notations introduites précédemment, la représentation $\pi_{\lambda}$ apparaît dans la représentation quasi-régulière de $G$ dans $L_{\mathbb{C}}^{2}(\Gamma \backslash G)$ si et seulement si, $\lambda\left(\log \Gamma \cap \mathfrak{g}_{\lambda}\right) \subset \mathbb{Z}$. La représentation $\pi_{\lambda}$ apparaît alors avec la multiplicité $m_{\lambda}=1$ si $\lambda\left(\mathfrak{g}^{\prime}\right)=\{0\}, m_{\lambda}=\left(\operatorname{det} \widetilde{B}_{\lambda}\right)^{1 / 2}$ si $\lambda\left(\mathfrak{g}^{\prime}\right) \neq\{0\}$, le déterminant étant calculé par rapport à n'importe quelle base de $\mathscr{L}_{\lambda}$.

10. Remarque. La condition apparaissant dans la proposition a bien un sens puisque si $\mu$ est dans l'orbite de $\lambda, \mu=\lambda+\lambda \circ \operatorname{ad}_{X}$ où $X \in \mathfrak{g}$. On en déduit que $B_{\lambda}=B_{\mu}$, donc $\mathfrak{g}_{\lambda}=\mathfrak{g}_{\mu}$, et que $\lambda$ et $\mu$ coïncident sur $\mathfrak{g}_{\lambda}$.

Preuve de la Proposition 9. Si la restriction de $\lambda$ à $\mathfrak{g}^{\prime}$ est nulle, la sous-algèbre maximale qui polarise $\lambda$ est $\mathfrak{g}$ et la condition ci-dessus est celle de la proposition A.3 (puisque $\mathfrak{g}_{\lambda}=\mathfrak{g}$ ). Supposons maintenant que la restriction de $\lambda$ à $\mathfrak{g}^{\prime}$ ne soit pas nulle est que la représentation $\pi_{\lambda}$ apparaissent dans la 
représentation quasi-régulière de $G$ dans $L_{\mathbb{C}}^{2}(\Gamma \backslash G)$. Quitte à changer $\lambda$ en $\lambda+\lambda \circ \operatorname{ad}_{X}(X \in \mathfrak{g})$, on peut supposer qu'il existe une sous-algèbre $\mathfrak{h}$ qui polarise $\lambda$ telle que $(\bar{\lambda}, H)$ soit un point entier (on a posé $H=\exp (\mathfrak{h})$ et $\bar{\lambda}$ est le caractère défini sur $H$ à l'aide de $\lambda$ ). Comme une sous-algèbre qui polarise $\lambda$ contient $\mathfrak{g}_{\lambda}$, on a forcément $\lambda\left(\mathfrak{g}_{\lambda} \cap \log \Gamma\right) \subset \mathbb{Z}$ (puisque $\bar{\lambda}(H \cap \Gamma$ ) $=1)$. Réciproquement, supposons que $\lambda$ soit tel que $\lambda\left(g_{\lambda} \cap \log \Gamma\right) \subset \mathbb{Z}$. Si $X, Y \in \log \Gamma$, alors $[X, Y] \in \log \Gamma$ (puisque $[\exp (X), \exp (Y)]=\exp ([X, Y]))$, donc $B_{\lambda}(X, Y) \in \mathbb{Z}$ puisque $\mathfrak{g}^{\prime} \subset \mathfrak{g}_{\lambda}$. On peut donc construire une base de $\mathfrak{g}\left\{U_{1}, \ldots, U_{k}, V_{1}, \ldots V_{k}, W_{1}, \ldots, W_{l}\right\} \quad\left(l=\operatorname{dim} \mathfrak{g}_{\lambda}\right.$ et $\left.2 k+l=\operatorname{dim} \mathfrak{g}\right)$ et des entiers $r_{1}, \ldots, r_{k}$ comme dans le lemme A4. Alors $\mathfrak{h}=\mathbb{R} W_{1} \oplus \cdots \oplus$ $\mathbb{R} W_{l} \oplus \mathbb{R} V_{1} \oplus \cdots \oplus \mathbb{R} V_{k}$ est une sous-algèbre rationnelle qui polarise $\lambda$. Notons $H=\exp (\mathfrak{h})$ et $\bar{\lambda}$ le caractère défini par $\bar{\lambda}(\exp (X))=\exp (2 \pi i \lambda(X))$ où $X \in \mathfrak{h}$; il suffit de montrer qu'il existe un point entier dans l'orbite du couple $(\bar{\lambda}, H)$. Rappelons que l'action de $G$ est donnée par $g .(\bar{\lambda}, H)=\left(\bar{\lambda} \circ I_{g}, I_{g^{-1}}(H)\right)$ où $I_{g}$ est l'automorphisme intérieur $y \mapsto g y g^{-1}(g, y \in G)$. Comme $\mathfrak{h}$ contient l'algèbre dérivée, $\mathfrak{h}$ est un idéal et $H$ est un sous-groupe normal de $G$, on en déduit que $g \cdot(\bar{\lambda}, H)=\left(\bar{\lambda} \circ I_{g}, H\right)$. Ecrivons $g$ et $\lambda$ sous la forme suivante:

$$
\begin{gathered}
g=\prod_{i=1}^{k} \exp \left(x_{i} U_{i}\right) \prod_{i=1}^{k} \exp \left(y_{i} V_{i}\right) \prod_{j=1}^{l} \exp \left(z_{j} V_{j}\right), \\
\lambda\left(\sum_{i=1}^{k}\left(u_{i} U_{i}+v_{i} V_{i}\right)+\sum_{j=1}^{l} w_{j} W_{j}\right)=\sum_{i=1}^{k}\left(u_{i} \alpha_{i}+v_{i} \beta_{i}\right)+\sum_{j=1}^{l} w_{j} \eta_{j} .
\end{gathered}
$$

Posons $X=\log g$ et remarquons que

$H \cap \Gamma=\left\{\prod_{i=1}^{k} \exp \left(t_{i} V_{i}\right) \prod_{j=1}^{l} \exp \left(s_{j} W_{j}\right)\right.$ où $\left(t_{1}, \ldots, t_{k}\right) \in \mathbb{Z}^{k}$ et $\left.\left(s_{1}, \ldots, s_{l}\right) \in \mathbb{Z}^{l}\right\}$.

Un calcul facile montre que

$$
\begin{aligned}
\bar{\lambda} \circ I_{g} & \left(\prod_{i=1}^{k} \exp \left(t_{i} V_{i}\right) \prod_{j=1}^{l} \exp \left(s_{j} W_{j}\right)\right) \\
& =\bar{\lambda}\left(\prod_{p=1}^{k} \exp \left(t_{p} V_{p}+\left[X, t_{p} V_{p}\right]\right) \prod_{q=1}^{l} \exp \left(s_{q} W_{q}+\left[X, s_{q} W_{q}\right]\right)\right) \\
& =\exp \left(2 \pi i \lambda\left(\sum_{p=1}^{k} t_{p} V_{p}+\left[X, t_{p} V_{p}\right]+\sum_{q=1}^{l} s_{q} W_{q}+\left[X, s_{q} W_{q}\right]\right)\right) \\
& =\exp \left(2 \pi i\left(\sum_{p=1}^{k} t_{p}\left(\alpha_{p}+r_{p} x_{p}\right)+\sum_{q=1}^{l} \eta_{q} s_{q}\right)\right) .
\end{aligned}
$$

Comme, par hypothèse, $\lambda\left(\log \Gamma \cap \mathfrak{g}_{\lambda}\right) \subset \mathbb{Z}, \eta_{q} \in \mathbb{Z}$ pour $q=1, \ldots, l$. Donc, pour que le couple $\left(\bar{\lambda} \circ I_{g}, H\right)$ soit un point entier, il faut et il suffit que $\alpha_{p}+$ $r_{p} x_{p} \in \mathbb{Z}$ pour $p=1, \ldots, k$. En particulier, si l'on choisit $x_{p}=-\alpha_{p} / r_{p}$ pour $p=1, \ldots, k,\left(\bar{\lambda} \circ I_{g}, H\right)$ sera un point entier et la représentation $\pi_{\lambda}$ 
apparaîtra dans la représentation quasi-régulière de $G$ dans $L_{\mathbb{C}}^{2}(\Gamma \backslash G)$, ce qui achève la preuve. Remarquons que l'on peut aussi calculer la multiplicité avec laquelle $\pi_{\lambda}$ apparaît. En effet, l'orbite de $(\bar{\lambda}, H)$ est l'ensemble des couples $\left\{\left(\chi_{\beta^{\prime}}, H\right)\right\}_{\beta^{\prime} \in \mathbb{R}^{k}}$ où $\chi_{\beta^{\prime}}$ est le caractère de $H$ défini par

$$
\chi_{\beta^{\prime}}\left(\prod_{p=1}^{k} \exp \left(s_{p} V_{p}\right) \prod_{q=1}^{l} \exp \left(t_{q} W_{q}\right)\right)=\exp \left(2 \pi i\left\{\sum_{p=1}^{k} \beta_{q}^{\prime} s_{p}+\sum_{q=1}^{l} \eta_{q} t_{q}\right\}\right) .
$$

De plus, le calcul effectué précédemment montre que le couple $\left(\chi_{\beta^{\prime}}, H\right)$ est un point entier si et seulement si $\beta^{\prime} \in \mathbb{Z}^{k}$ et que deux points entiers $\left(\chi_{\beta^{\prime}}, H\right)$ et $\left(\chi_{\beta^{\prime \prime}}, H\right)$ sont dans la même $\Gamma$-orbite si et seulement si $\beta_{i}^{\prime} \equiv \beta_{i}^{\prime \prime} \bmod r_{i}$ pour $i=1, \ldots, k$. On en déduit que la représentation $\pi_{\lambda}$ apparaît avec multiplicité $r_{1} \cdots r_{k}$.

Il est clair que les images des vecteurs $U_{1}, \ldots, U_{k}, V_{1}, \ldots, V_{k}$ forment une base de $\mathscr{L}_{\lambda}$, donc

$$
\operatorname{det} \widetilde{B}_{\lambda}=\left(\operatorname{det}\left(B_{\lambda}\left(U_{i}, V_{j}\right)\right)_{1 \leq i, j \leq k}\right)^{2}=\left(\prod_{i=1}^{k} r_{i}\right)^{2} .
$$

Pour terminer cette partie, on va voir que dans certains cas, la description de l'ensemble $\mathscr{A}(\Gamma)$ peut être simplifiée.

11. Définition. Soit $G$ un groupe de Lie nilpotent simplement connexe, un sous-groupe uniforme $\Gamma$ de $G$ est appelé un réseau si $\log \Gamma$ est un sous-groupe de $\mathfrak{g}$.

Un résultat, dû Moore [14], assure que si $\Gamma$ est un sous-groupe uniforme d'un groupe de Lie nilpotent simplement connexe, il existe deux réseaux $\Gamma_{1}$ et $\Gamma_{2}$ tels que $\Gamma_{1} \subset \Gamma \subset \Gamma_{2}$ et que $\Gamma_{1}$ soit d'indice fini dans $\Gamma_{2}$. Dans le cas où $\Gamma$ est un réseau, on peut utiliser la proposition A.9 pour retrouver un résultat connu [14].

12. Proposition. Soient $G$ un groupe de Lie nilpotent simplement connexe de rang deux et $\Gamma$ un réseau de $G$, une orbite $\mathscr{O}$ est adaptée à $\Gamma$ si et seulement si il existe $\lambda \in \mathscr{O}$ tel que $\lambda(\log \Gamma) \subset \mathbb{Z}$.

Preuve. Il est clair que si $\lambda \in \mathfrak{g}^{*}$ est tel que $\lambda(\log \Gamma) \subset \mathbb{Z}$, alors son orbite $\mathscr{O}$ est adaptée à $\Gamma$. Réciproquement, soit $\lambda \in \mathfrak{g}^{*}$ tel que $\lambda\left(\log \Gamma \cap \mathfrak{g}_{\lambda}\right) \subset \mathbb{Z}$. Si la restriction de $\lambda$ à $\mathfrak{g}^{\prime}$ est nulle, alors $\mathfrak{g}_{\lambda}=\mathfrak{g}$ et $\lambda(\log \Gamma) \subset \mathbb{Z}$. Supposons maintenant que la restriction de $\lambda$ à $\mathfrak{g}^{\prime}$ ne soit pas nulle; d'après ce que l'on a vu précédemment la forme $B_{\lambda}$ prend des valeurs entières sur $\log \Gamma$ et il existe une base de $\log \Gamma,\left\{U_{1}, \ldots, U_{k}, V_{1}, \ldots, V_{k}, W_{1}, \ldots, W_{l}\right\}$ et des entiers $r_{1}, \ldots, r_{k}$ comme dans le Lemme 4 . Ecrivons $\lambda$ sous la forme

$$
\lambda\left(\sum_{i=1}^{k}\left(u_{i} U_{i}+v_{i} V_{i}\right)+\sum_{j=1}^{l} w_{j} W_{j}\right)=\sum_{i=1}^{k}\left(\alpha_{i} u_{i}+\beta_{i} v_{i}\right)+\sum_{j=1}^{l} \eta_{j} w_{j}
$$

Par hypothèse, $\eta_{j} \in \mathbb{Z}$ pour $j=1, \ldots, l$. Si $X=\sum_{i=1}^{k}\left(x_{i} U_{i}+y_{i} V_{i}\right)+$ $\sum_{j=1}^{l} z_{j} W_{j}$, alors $\mu=\lambda+\lambda \circ \operatorname{ad}_{X}$ est dans d'orbite de $\lambda$ et un calcul facile 
montre que

$\mu\left(\sum_{i=1}^{k}\left(u_{i} U_{i}+v_{i} V_{i}\right)+\sum_{j=1}^{l} w_{j} W_{j}\right)=\sum_{i=1}^{k}\left[\left(\alpha_{i}-y_{i} r_{i}\right) u_{i}+\left(\beta_{i}+x_{i} r_{i}\right) v_{i}\right]+\sum_{j=1}^{l} \eta_{j} w_{j}$

Donc, si l'on choisit $y_{i}=\alpha_{i} / r_{i}$ et $x_{i}=-\beta_{i} / r_{i}$ pour $i=1, \ldots, k$, on aura bien $\mu(\log \Gamma) \subset \mathbb{Z}$.

13. Remarque. D'après le calcul qui vient dêtre fait, si $\lambda$ et $\mu$ sont deux formes linéaires, $\mu$ est dans l'orbite de $\lambda$ si et seulement si $\mu$ a même restriction que $\lambda$ à $\mathfrak{g}_{\lambda}$.

\section{APPENDICE B}

Dans cet appendice, on étudie deux cas particuliers et on redémontre la Proposition III.7, dans ces cas particuliers, en utilisant une techrique un peu différente.

Exemple I. Soient $n$ et $p$ deux entiers strictement positifs. Notons $G_{n, p}$ le groupe de Lie difféomorphe à $\mathbb{R}^{2 n+1+p}$ dont la loi de groupe est donnée par

$$
(x, y, w, z) \cdot\left(x^{\prime}, y^{\prime}, w^{\prime}, z^{\prime}\right)=\left(x+x^{\prime}, y+y^{\prime}, w+w^{\prime}, z+z^{\prime}+\left\langle x, y^{\prime}\right\rangle\right)
$$

où $x, y, x^{\prime}, y^{\prime} \in \mathbb{R}^{n} ; w, w^{\prime} \in \mathbb{R}^{p} ; z, z^{\prime} \in \mathbb{R}$ et $\langle\cdot, \cdot\rangle$ désigne le produit scalaire usuel sur $\mathbb{R}^{n}$. $G_{n, p}$ est le produit direct du groupe de Heisenberg $H_{n}=\left\{(x, y, 0, z) \in G_{n, p}\right\}$ et de $\mathbb{R}^{p}$. On vérifie facilement que l'algèbre de Lie de $G_{n, p}$ est $\mathfrak{g}_{n, p}=\mathbb{R}^{2 n} \oplus \mathbb{R}^{p} \oplus \mathbb{R}$ où, si $\left\{X_{1}, \ldots, X_{n}, Y_{1}, \ldots, Y_{n}\right\}$ (resp. $\left.\left\{W_{1}, \ldots, W_{p}\right\} ;\{Z\}\right)$ désigne la base canonique de $\mathbb{R}^{2 n}\left(\right.$ resp. $\mathbb{R}^{p} ; \mathbb{R}$ ), les seuls crochets non nuls entre les éléments de la base $\mathscr{B}=\left\{X_{1}, \ldots, X_{n}\right.$, $\left.Y_{1}, \ldots, Y_{n}, W_{1}, \ldots, W_{p}, Z\right\}$ sont $\left[X_{i}, Y_{i}\right]=Z$ pour $i=1, \ldots, n$. On en déduit que pour tout $X, Y \in \mathbb{R}^{2 n} \oplus \mathbb{R}^{p}=\mathbb{R}^{2 n+p},[X, Y]=A(X, Y) Z$ où $A$ est la forme bilinéaire antisymétrique dont la matrice par rapport à la base $\left\{X_{1}, \ldots, X_{n}, Y_{1}, \ldots, Y_{n}, W_{1}, \ldots, W_{p}\right\}$ est

$$
J_{n, p}=\left(\begin{array}{ccc}
\mathscr{O}_{n} & I_{n} & 0 \\
-I_{n} & \mathscr{O}_{n} & 0 \\
0 & 0 & \mathscr{O}_{p}
\end{array}\right)
$$

On vérifie que $\mathfrak{g}_{n, p}$ est une algèore de Lie nilpotente de rang deux dont le centre est $\mathfrak{z}=\mathbb{R}^{p} \oplus \mathbb{R} Z$ et l'algèbre dérivée est $\mathfrak{g}_{n, p}^{\prime}=\mathbb{R} Z$. D'autre part, on voit facilement que l'application exponentielle est donnée par $\exp ((x, y, w, z))=$ $\left(x, y, w, z+\frac{1}{2}\langle x, y\rangle\right)$ pour $x, y \in \mathbb{R}^{n} ; w \in \mathbb{R}^{p}$ et $z \in \mathbb{R}$.

On va tout d'abord classifier les sous-groupes uniformes de $G_{n, p}$. Pour cela, on introduit l'ensemble $\mathscr{D}_{n}=\left\{r=\left(r_{1}, \ldots, r_{n}\right) \in\left(\mathbb{N}^{*}\right)^{n}\right.$ tels que $r_{i}$ divise $r_{i+1}$ pour $i=1, \ldots, n-1\}$ et on note $\delta_{r}$ la matrice carrée diagonale d'ordre $2 n+p$ dont les coefficients diagonaux sont $r_{1}, \ldots, r_{n}, \underbrace{1, \ldots, 1}_{(n+p \text { fois })}$. Soit

$$
\Gamma_{r}=\left\{(x, y, w, z) \in G_{n, p} \text { tel que }\left(\begin{array}{c}
x \\
y \\
w
\end{array}\right) \in \delta_{r} \mathbb{Z}^{2 n+p}, z \in \mathbb{Z}\right\},
$$

on vérifie facilement que $\Gamma_{r}$ est un sous-groupe uniforme de $G_{n, p}$. En fait, tous les sous-groupes uniformes de $G_{n, p}$ sont de cette forme. De manière plus précise, en raisonnant comme dans [7, Théorème 2.4], on montre 
1. Proposition. Soit $\Gamma$ un sous-groupe uniforme de $G_{n, p}$, il existe $\varphi \in$ $\operatorname{Aut}\left(G_{n, p}\right)$ et $r \in \mathscr{D}_{n}$ tels que $\varphi(\Gamma)=\Gamma_{r}$. De plus, si $r \neq s \quad\left(r, s \in \mathscr{D}_{n}\right)$ les groupes $\Gamma_{r}$ et $\Gamma_{s}$ ne sont pas isomorphes en tant que groupe abstrait (en particulier, les variétés $\Gamma_{r} \backslash G_{n, p}$ et $\Gamma_{s} \backslash G_{n, p}$ ne sont pas homéomorphes).

On va maintenant voir que, comme dans le cas du groupe de Heisenberg $H_{n}$ [16], on ne peut pas construire de déformations isospectrales non triviales à l'aide de la proposition III.3. En effet.

2. Lemme. Soit $\Gamma$ un sous-groupe unfiorme de $G_{n, p}$, alors $\operatorname{AIA}\left(G_{n, p} ; \Gamma\right)=$ $\operatorname{Inn}\left(G_{n, p}\right)$.

Preu:'e. Il suffit de démontrer le lemme dans le cas $\Gamma=\Gamma_{r}$ où $r \in \mathscr{D}_{n}$ et, comme les groupes considérés sont simplement connexes, il suffit de démontrer que $\operatorname{AID}\left(\mathfrak{g}, \Gamma_{r}\right)=\operatorname{Inn}(\mathfrak{g})$. Soit $D \in \operatorname{AID}\left(\mathfrak{g} ; \Gamma_{r}\right)$, alors $D\left(r_{i} X_{i}\right) \in\left[\mathfrak{g}, r_{i} X_{i}\right]$ et $D\left(Y_{i}\right) \in\left[\mathfrak{g}, Y_{i}\right] \quad(i=1, \ldots, n) ; D\left(W_{j}\right) \in\left[\mathfrak{g}, W_{j}\right](j=1, \ldots, p)$ et $D(Z) \in[\mathfrak{g}, Z]$ puisque ces vecteurs sont dans $\log \Gamma$. On en déduit que $D(Z)=$ $D\left(W_{j}\right)=0(j=1, \ldots, p)$ et qu'il existe des réels $\alpha_{i}$ et $\beta_{j}(i=1, \ldots, n)$ tels que $D\left(X_{i}\right)=\alpha_{i} Z$ et $D\left(Y_{i}\right)=\beta_{i} Z$. Donc, si l'on pose $X=\sum_{i=1}^{n} \beta_{i} X_{i}-$ $\alpha_{i} Y_{i}, D=\operatorname{ad}_{X}$. On en déduit que $\operatorname{AID}\left(\mathfrak{g}_{n, p} ; \Gamma_{r}\right) \subset \operatorname{Inn}\left(\mathfrak{g}_{n, p}\right)$. L'inclusion $\operatorname{Inn}\left(\mathfrak{g}_{n, p}\right) \subset \operatorname{AID}(\mathfrak{g}, \Gamma)$ étant évidente, le lemme est démontré.

On peut maintenant démontrer ("rigidité spectrale"):

3. Proposition. Soient $\Gamma$ un sous-groupe uniforme de $G_{n, p},\left\{\mathbf{m}_{s}\right\}_{s \in I}$ une famille continue de métriques invariantes à gauche. Si les variétés $\left(\Gamma \backslash G_{n, p}, \mathbf{m}_{s}\right)$ sont deux à deux isospectrales, elles sont deux à deux isométriques.

Preuve. On va imposer deux normalisations successives. Tout d'abord, d'après le proposition B.1, il existe $\varphi \in \operatorname{Aut}\left(G_{n, p}\right)$ et $r \in \mathscr{D}_{n}$ tels que $\varphi(\Gamma)=\Gamma_{r}$. Comme les variétés $\left(\Gamma \backslash G_{n, p}, \mathbf{m}_{s}\right)$ et $\left(\Gamma_{r} \backslash G_{n, p},\left(\varphi^{-1}\right)^{*} \mathbf{m}_{s}\right)$ sont isométriques, si l'on pose $\mathbf{m}_{s}^{\prime}=\left(\varphi^{-1}\right)^{*} \mathbf{m}_{s},\left\{\mathbf{m}_{s}^{\prime}\right\}_{s \in I}$ est encore une famille continue de métriques invariantes à gauche (puisque $\varphi \in \operatorname{Aut}\left(G_{n, p}\right)$ et que, sur $\mathfrak{g}_{n, p}$,

$$
\left.\mathbf{m}_{s}^{\prime}={ }^{t}\left(\varphi_{*}^{-1}\right) \mathbf{m}_{s}\left(\varphi_{*}^{-1}\right)\right)
$$

qui est telle que les variétés $\left(\Gamma_{r} \backslash G_{n, p}, \mathbf{m}_{s}^{\prime}\right)$ sont deux à deux isospectrales $(s \in$ I). Définissons maintenant le vectuer $X_{s} \in \mathfrak{g}_{n, p}$ par

$$
X_{s}=\left(\sum_{i=1}^{n} \mathbf{m}_{s}^{\prime}\left(X_{i}, Z\right) Y_{i}-\mathbf{m}_{s}^{\prime}\left(Y_{i}, Z\right) X_{i}\right) / \mathbf{m}_{s}^{\prime}(Z, Z)
$$

et appelons $\varphi_{s}$ l'automorphisme intérieur tel que $\left(\varphi_{s}\right)_{*}=\mathrm{Id}+\operatorname{ad}_{X_{s}}$. Alors

$$
\left(\varphi_{s}\right)_{*}\left(X_{i}\right)=X_{i}-\mathbf{m}_{s}^{\prime}\left(X_{i}, Z\right) \mathbf{m}_{s}^{\prime}(Z, Z)^{-1} Z
$$

et

$$
\left(\varphi_{s}\right)_{*}\left(Y_{i}\right)=Y_{i}-\mathbf{m}_{s}^{\prime}\left(Y_{i}, Z\right) \mathbf{m}_{s}^{\prime}(Z, Z)^{-1} Z
$$

pour $i=1, \ldots, n ;\left(\varphi_{s}\right)_{*}\left(W_{j}\right)=W_{j}$ pour $j=1, \ldots, p$ et $\left(\varphi_{s}\right)_{*}(Z)=Z$. On en déduit que, si l'on pose $\mathbf{m}_{s}^{\prime \prime}=\left(\varphi_{s}\right)^{*} \mathbf{m}_{s}^{\prime}$ pour $s \in I$, alors:

(1) Pour tout $s \in I$, les variétés $\left(\Gamma_{r} \backslash G_{n, p}, \mathbf{m}_{s}^{\prime}\right)$ et $\left(\Gamma_{r} \backslash G_{n, p}, \mathbf{m}_{s}^{\prime \prime}\right)$ sont isométriques.

(2) La famille $\left\{\boldsymbol{m}_{s}^{\prime \prime}\right\}_{s \in I}$ est une famille de métriques invariantes à gauche qui est clairement continue (puisque l'application $s \mapsto \varphi_{s}$ est continue). 
(3) Pour tout $s \in I$, et $j \in\{1, \ldots, n\}, \mathbf{m}_{s}^{\prime \prime}\left(Z, X_{j}\right)=\mathbf{m}_{s}^{\prime \prime}\left(Z, Y_{j}\right)=0$.

Il résulte de (1) que les variétés $\left(\Gamma_{r} \backslash G_{n, p}, \mathbf{m}_{s}^{\prime \prime}\right)$ sont deux à deux isospectrales. La fin de la preuve va consister à montrer que l'application $s \mapsto \mathbf{m}_{s}^{\prime \prime}$ est constante. Soit $\psi$ l'automorphisme de $G_{n, p}$ tel que

$\psi_{*}\left(X_{i}\right)=X_{i} \quad$ et $\quad \psi_{*}\left(Y_{i}\right)=Y_{i} \quad$ pour $i=1, \ldots, n$;

$\psi_{*}\left(W_{j}\right)=W_{j}-\mathbf{m}_{s}^{\prime \prime}\left(W_{j}, Z\right) \mathbf{m}_{s}^{\prime \prime}(Z, Z)^{-1} Z$ pour $j=1, \ldots, p$;

$\psi_{*}(Z)=Z$.

D'après le proposition III.6, $\psi$ est indépendante de $s$ et si l'on pose $\mathbf{m}_{s}^{\prime \prime \prime}=$ $\psi^{*} \mathbf{m}_{s}^{\prime \prime}$, alors:

(a) Pour tout $s \in I$, les variétés $\left(\psi^{-1}\left(\Gamma_{r}\right) \backslash G_{n, p}, \mathbf{m}_{s}^{\prime \prime \prime}\right)$ et $\left(\Gamma_{r} \backslash G_{n, p}, \mathbf{m}_{s}^{\prime \prime}\right)$ sont isométriques, donc isospectrales.

(b) La famille $\left\{\mathbf{m}_{s}^{\prime \prime \prime}\right\}_{s \in I}$ est une famille continue de métriques invariantes à gauche.

(c) Pour tout $s \in I$ et pour tout $X \in \mathbb{R}^{2 n+p}=\mathbb{R}^{2 n} \oplus \mathbb{R}^{p}, \mathbf{m}_{s}^{\prime \prime \prime}(X, Z)=0$.

Soit $\pi:\left(\psi^{-1}\left(\Gamma_{r}\right) \backslash G_{n, p}, \mathbf{m}_{s}^{\prime \prime}\right) \rightarrow\left(\mathscr{L}_{r} \backslash \mathbb{R}^{2 n+p}, \mathbf{n}_{s}\right) \quad(x, y, q, z) \mapsto(x, y, w)$ où $\mathbf{n}_{s}$ est la restriction à $\mathbb{R}^{2 n+p}$ de $\mathbf{m}_{s}^{\prime \prime \prime}$ et $\mathscr{L}_{r}$ est le réseau $\delta_{r} \cdot \mathbb{Z}^{2 n+p}$. D'après la propriété (c), $\pi$ est une submersion riemannienne dont la base est un tore plat. De plus, comme le centre d'un groupe de Lie est totalement géodésique pour une métrique invariante à gauche (en effet, si $(\cdot \mid \cdot)$ est une métrique invariante à gauche et si $\nabla$ désigne la connexion associée, alors $\left(\nabla_{X} X \mid Y\right)=([Y, X] \mid X)$ pour $X, Y$ dans l'algèbre de Lie), on a une submersion riemannienne à fibres totalement géodésiques. Donc, si $\Sigma$ désigne le spectre commun aux variétés $\left(\Gamma \backslash G, \mathbf{m}_{s}\right)$, pour tout $s \in I$, le spectre du tore plat $\left(\mathscr{L}_{r} \backslash \mathbb{R}^{2 n+p}, \mathbf{n}_{s}\right)$ est contenu dans $\Sigma$ [1]. Comme l'application $s \mapsto \mathbf{n}_{s}$ est continue, on en déduit que cette application est constante. Donc pour tout $s \in I, \mathbf{n}_{s}=\mathbf{n}_{s_{0}}$. Or si $X, Y \in \mathbb{R}^{2 n}$,

$$
\mathbf{m}_{s}^{\prime \prime}(X, Y)=\mathbf{m}_{s}^{\prime \prime \prime}(X, Y)=\mathbf{m}_{s_{0}}^{\prime \prime}(X, Y)=\mathbf{m}_{s_{0}}^{\prime \prime}(X, Y)
$$

et si $X \in \mathbb{R}^{2 n}$ et $W \in \mathbb{R}^{p}$,

$$
\mathbf{m}_{s}^{\prime \prime}(X, W)=\mathbf{m}_{s}^{\prime \prime \prime}(X, W)=\mathbf{m}_{s_{0}}^{\prime \prime \prime}(X, W)=\mathbf{m}_{s_{0}}^{\prime \prime}(X, W)
$$

(puisque $\mathbf{m}_{s}^{\prime \prime}(X, Z)=0$ pour $X \in \mathbb{R}^{2 n}$ ). Comme la restriction de $\mathbf{m}_{s}^{\prime \prime}$ au centre est indépendante de $s$ on en déduit que pour tout $s \in I, \mathbf{m}_{s}^{\prime \prime}=\mathbf{m}_{s_{0}}^{\prime \prime}$. Comme les variétés $\left(\Gamma \backslash G_{n, p}, \mathbf{m}_{s}\right)$ et $\left(\Gamma_{1} \backslash G_{n, p}, \mathbf{m}_{s}^{\prime \prime}\right)$ sont isométriques, la proposition est démontrée.

Exemple II. Soit $\mathfrak{g}$ l'algèbre de Lie de dimension 6 ayant comme base $\mathscr{B}=$ $\left\{X_{1}, X_{2}, Y_{1}, Y_{2}, Z_{1}, Z_{2}\right\}$, les crochets non nuls entre les éléments de $\mathscr{B}$ étant: $\left[X_{1}, Y_{1}\right]=\left[X_{2}, Y_{2}\right]=Z_{1},\left[X_{1}, Y_{2}\right]=Z_{2}$. Alors $\mathfrak{g}$ peut être vue comme l'algèbre de Lie du groupe de matrices suivant:

$$
G=\left\{\sigma\left(x_{1}, x_{2}, y_{1}, y_{2}, z_{1}, z_{2}\right)=\left(\begin{array}{ccccccc}
1 & x_{1} & x_{2} & z_{1} & & & \\
0 & 1 & 0 & y_{1} & & 0 & \\
0 & 0 & 1 & y_{2} & & & \\
0 & 0 & 0 & 1 & & & \\
& & & & 1 & x_{1} & z_{2} \\
& 0 & & & 0 & 1 & y_{2} \\
& & & & 0 & 0 & 1
\end{array}\right)\right\}
$$

où $\left(x_{1}, x_{2}, y_{1}, y_{2}, z_{1}, z_{2}\right) \in \mathbb{R}^{6}$. On vérifie facilement que la loi de groupe est 
donnée par

$$
\begin{aligned}
& \sigma\left(x, y, z_{1}, z_{2}\right) \cdot \sigma\left(x^{\prime}, y^{\prime}, z_{1}^{\prime}, z_{2}^{\prime}\right) \\
& \quad=\sigma\left(x+x^{\prime}, y+y^{\prime}, z_{1}+z_{1}^{\prime}+\left\langle x, y^{\prime}\right\rangle, z_{2}+z_{2}^{\prime}+x_{1} y_{2}^{\prime}\right)
\end{aligned}
$$

où $x=\left(x_{1}, x_{2}\right), y=\left(y_{1}, y_{2}\right), x^{\prime}=\left(x_{1}^{\prime}, x_{2}^{\prime}\right), y^{\prime}=\left(y_{1}^{\prime}, y_{2}^{\prime}\right)$ sont des éléments de $\mathbb{R}^{2}$ et où $\langle\cdot, \cdot\rangle$ désigne le produit scalaire usuel dans $\mathbb{R}^{2}$ et que

$$
\exp ((x, y, z))=\sigma\left(x, y, z_{1}+\frac{1}{2}\langle x, y\rangle, z_{2}+\frac{1}{2} x_{1} y_{2}\right)
$$

où l'on a posé

$$
(x, y, z)=x_{1} X_{1}+x_{2} X_{2}+y_{1} Y_{1}+y_{2} Y_{2}+z_{1} Z_{1}+z_{2} Z_{2} .
$$

On vérifie facilement que $\mathfrak{g}$ est une algèbre de Lie nilpotente de rang deux dont le centre $\mathfrak{z}$ est $\mathbb{R} Z_{1} \oplus \mathbb{R} Z_{2}$ et dont l'algèbre dérivée $\mathfrak{g}^{\prime}$ est égale à $\mathfrak{z}$.

On se contentera ici d'étudier le cas où $\Gamma$ est le sous-groupe de $G$ constitué des matrices à coefficients entiers. Un calcul facile [6], montre qu'un endomorphisme $D$ de $\mathfrak{g}$ est un élément de $\operatorname{AID}(\mathfrak{g}, \Gamma)$ si et seulement si

(1) $\operatorname{Im}(D) \subset \mathfrak{z}$;

(2) $D\left(X_{2}\right) \in \mathbb{R} Z_{1} ; D\left(Y_{1}\right) \in \mathbb{R} Z_{1}$.

On en déduit que $\operatorname{dim} \operatorname{AID}(\mathfrak{g} ; \Gamma)=6$. Comme $\operatorname{dim} \operatorname{Inn}(\mathfrak{g})=\operatorname{dim} \mathfrak{g} / \mathfrak{z}=$ $4, \operatorname{AIA}(G ; \Gamma) \neq \operatorname{Inn}(G)$ et on vérifie facilement que les deux dérivations $D_{1}$ et $D_{2}$ qui sont telles que $D_{1}\left(X_{1}\right)=D_{2}\left(Y_{2}\right)=Z_{1}$ et qui sont nulles sur les autres vecteurs de la base $\mathscr{B}$ engendrent un supplémentaire de $\operatorname{Inn}(\mathfrak{g})$ dans $\operatorname{AID}(\mathfrak{g} ; \Gamma)$. On peut alors montrer qu'il existe des déformations isospectrales non triviales de la forme $\left(\Gamma \backslash G, \mathbf{m}_{s}\right)$ où $\mathbf{m}_{s}$ est de la forme $\mathbf{m}_{s}=\varphi_{s}^{*} \mathbf{m}_{s}$ où $\varphi_{s} \in \operatorname{AIA}(G ; \Gamma)[4,6]$. De plus, De Turck, Gluck, Gordon, et Webb ont étudié le comportement de la "masse" d'une classe d'homologie lors d'une telle déformation [3]. On va voir, qu'en fait, toutes les déformations isospectrales sont de ce type, comme c'est le cas pour les groupes de Lie nilpotent simplement connexe non singuliers et pour les groupes $G_{n, p}$. En effet:

4. Proposition. Soient $G$ et $\Gamma$ comme ci-dessus, soient $\left\{\mathbf{m}_{s}\right\}_{s \in I}$ une famille continue de métriques invariantes à gauche. Si les variétés $\left(\Gamma \backslash G, \mathbf{m}_{s}\right)$ sont deux à deux isospectrales, il existe une famille continue $\left\{\psi_{s}\right\}_{s \in I}$ d'éléments de $\operatorname{AIA}(G ; \Gamma)$ telle que pour tout $s \in I$, on ait $\mathbf{m}_{s}=\psi_{s}^{*} \mathbf{m}_{s_{0}},\left(s_{0} \in I\right)$.

Preuve. On va tout d'abord, comme dans le cas de $G_{n, p}$, construire, à l'aide d'éléments de $\operatorname{AIA}(G ; \Gamma)$, une famille continue $\left\{\mathbf{m}_{s}^{\prime}\right\}_{s \in I}$ de métriques invariantes à gauche répondant à certaines conditions de normalisation. Pour cela remarquons que pour tout $s \in I$, il existe un unique élément $X_{1}^{\prime}(s) \in \mathfrak{z}$ tel que $\mathbf{m}_{s}\left(X_{1}+X_{1}^{\prime}(s), Z\right)=0$ (en effet, l'application $Z \mapsto \mathbf{m}_{s}\left(X_{1}, Z\right)$ est dans $\mathfrak{z}^{*}$, ce qui assure l'existence et l'unicité d'un tel élément). De plus, on vérifie facilement que l'application $s \mapsto X_{1}^{\prime}(s)$ est continue. De même, on démontre l'existence d'une application continue de $I$ dans $\mathfrak{z}, s \mapsto Y_{2}^{\prime}(s)$ telle que pour tout $Z \in \mathfrak{z}$, $\mathbf{m}_{s}\left(Y_{2}+Y_{2}^{\prime}(s), Z\right)=0$. Comme l'application $\operatorname{AID}(\mathfrak{g} ; \Gamma) \rightarrow \mathfrak{z} \oplus \mathbb{R} Z_{1} \oplus \mathbb{R} Z_{2} \oplus \mathfrak{z}$, $D \mapsto\left(D\left(X_{1}\right), D\left(X_{2}\right), D\left(Y_{1}\right), D\left(Y_{2}\right)\right)$ est un isomorphisme d'espaces vectoriels (puisque cette application est injective et que les deux espaces sont de dimension 6), il existe un famille continue $\left\{D_{s}\right\}_{s \in I}$ d'éléments de $\operatorname{AID}(\mathfrak{g} ; \Gamma)$ telle 
que pour tout $s \in I$, on ait

$$
\begin{aligned}
& D_{s}\left(X_{1}\right)=X_{1}^{\prime}(s) ; \\
& D_{s}\left(Y_{2}\right)=Y_{2}^{\prime}(s) ; \\
& D_{s}\left(X_{2}\right)=-\mathbf{m}_{s}\left(X_{2}, Z_{1}\right) \mathbf{m}_{s}\left(Z_{1}, Z_{1}\right)^{-1} Z_{1} ; \\
& D_{s}\left(Y_{1}\right)=-\mathbf{m}_{s}\left(Y_{1}, Z_{1}\right) \mathbf{m}_{s}\left(Z_{1}, Z_{1}\right)^{-1} Z_{1} .
\end{aligned}
$$

Soit $\varphi_{s}$ l'élément de $\operatorname{AIA}(G ; \Gamma)$ tel que $\left(\varphi_{s}\right)_{*}=\operatorname{Id}+D_{s}$; alors la famille de métriques $\left\{\mathbf{m}_{s}^{\prime}\right\}_{s \in I}$ définie par $\mathbf{m}_{s}^{\prime}=\varphi_{s}^{*} \mathbf{m}_{s}$ est une famille continue de métriques invariantes à gauche telles que pour tout $s \in I$ :

(1) Les variétés $\left(\Gamma \backslash G, \mathbf{m}_{s}\right)$ et $\left(\Gamma \backslash G, \mathbf{m}_{s}^{\prime}\right)$ sont isospectrales. $=0$.

(2) Pour tout $Z \in \mathfrak{z}, \mathbf{m}_{s}^{\prime}\left(X_{1}, Z\right)=\mathbf{m}_{s}^{\prime}\left(Y_{2}, Z\right)=0, \mathbf{m}_{s}^{\prime}\left(X_{2}, Z_{1}\right)=\mathbf{m}_{s}^{\prime}\left(Y_{1}, Z_{1}\right)$

Pour terminer la preuve, il suffit de montrer que l'application $s \mapsto \mathbf{m}_{s}^{\prime}$ est constante. En effet, si pour tout $s \in I, \mathbf{m}_{s}^{\prime}=\mathbf{m}_{s_{0}}^{\prime}\left(s_{0} \in I\right)$ on aura $\mathbf{m}_{s}=$ $\left(\varphi_{s_{0}} \varphi_{s}^{-1}\right)^{*} \mathbf{m}_{s_{0}}$, d'où le résultat voulu avec $\psi_{s}=\varphi_{s_{0}} \varphi_{s}^{-1}$. Montrons donc que l'application $s \mapsto \mathbf{m}_{s}^{\prime}$ est constante. Soit $\lambda_{0}$ dans $\mathfrak{g}^{*}$ tel que $\lambda_{0}\left(Z_{1}\right)=0$ et $\lambda_{0}\left(Z_{2}\right) \neq 0$, un calcul facile montre que $\mathfrak{g}_{\lambda_{0}}=\mathbb{R} X_{2} \oplus \mathbb{R} Y_{1} \oplus \mathbb{R} Z_{1} \oplus \mathbb{R} Z_{2}$ et que l'orbite de $\lambda_{0}$ est l'ensemble des éléments de $\mathfrak{g}^{*}$ qui coïncident avec $\lambda_{0}$ sur $\mathfrak{g}_{\lambda_{0}}$. D'autre part, un tel $\lambda \in \mathfrak{g}^{*}$ est adapté à $\Gamma$ si $\lambda\left(\log \Gamma \cap \mathfrak{g}_{\lambda_{0}}\right) \subset \mathbb{Z}$, c'està-dire si $\lambda\left(X_{2}\right), \lambda\left(Y_{1}\right)$ et $\lambda\left(Z_{2}\right)$ sont des entiers (rappelons que $\lambda\left(Z_{2}\right) \neq 0$ ). Notons $\mathbf{n}_{s}$ la restriction de $\mathbf{m}_{s}^{\prime}$ à $\mathfrak{g}_{\lambda_{0}}$, alors, en raisonnant comme dans la proposition III.6, on prouve que si $\left\{U_{i}(s)\right\}_{1<i<4}$ est une base orthonormée de $\mathfrak{g}_{\lambda_{0}}$ pour la métrique $\mathbf{n}_{s}, \sum_{i=1}^{4}\left(\lambda\left(U_{i}(s)\right)\right)^{2}=\sum_{i=1}^{4}\left(\lambda\left(U_{i}\left(s_{0}\right)\right)\right)^{2}$ pour tout $s \in I$ et pour tout $\lambda$ adapté à $\Gamma$ tel que $\lambda\left(Z_{1}\right)=0$ et $\lambda\left(Z_{2}\right) \neq 0$. Fixons la base $\mathscr{B}^{\prime}=\left\{X_{2}, Y_{1}, Z_{2}, Z_{1}\right\}$ de $\mathfrak{g}_{\lambda_{0}}$ et identifions $\mathbb{R}^{3}$ avec le sous-espace $\mathbb{R} X_{2} \oplus \mathbb{R} Y_{1} \oplus \mathbb{R} Z_{2}$ de $\mathfrak{g}_{\lambda_{0}}$. Soit $\mathscr{A}=\left\{(p, s, r) \in \mathbb{Z}^{3}\right.$ tels que $\left.r \neq 0\right\}$, d'après ce que l'on vient de voir, pour tout $x \in \mathscr{A}$ et pour tout $s \in I,{ }^{t} x \mathbf{n}_{s}^{-1}={ }^{t} x \mathbf{n}_{s_{0}}^{-1} x$ $\left(s_{0} \in I\right)$. Comme l'ensemble $\{\alpha x ; \alpha \in \mathbb{R}$ et $x \in \mathscr{A}\}$ est dense dans $\mathbb{R}^{3}$, on en déduit que pour tout $x \in \mathbb{R}^{3}$ et pour tout $s \in I,{ }^{t} x \mathbf{n}_{s}^{-1} x={ }^{t} x \mathbf{n}_{s_{0}}^{-1} x$. Il existe donc une forme linéaire $\nu_{0} \in\left(\mathbb{R}^{3}\right)^{*}$ telle que pour tout $x \in \mathbb{R}^{3}$, $\mathbf{m}_{s}^{-1} x=\mathbf{n}_{s_{0}}^{-1} x+\nu_{s}(x) Z_{1}$, et ceci pour tout $s \in I$. Or $\mathbf{n}_{s_{0}} X_{2} \in \mathbb{R}^{3}$, donc $\mathbf{n}_{s_{0}} X_{2}=\mathbf{n}_{s} X_{2}+\nu_{s}\left(\mathbf{n}_{s_{0}} X_{2}\right) \mathbf{n}_{s} Z_{1}$. En multipliant les deux membres de cette égalité par ${ }^{t} Z_{1}$ à gauche, on troue que $\nu_{s}\left(\mathbf{n}_{s}, Z_{1}\right)=0$, donc $\mathbf{n}_{s_{0}} X_{2}=\mathbf{n}_{s} X_{2}$. On démontre de même que pour tout $s \in I, \mathbf{n}_{s} Y_{1}=\mathbf{n}_{s_{0}} Y_{1}$. Comme on sait que la restriction de $\mathbf{n}_{s}$ au centre est indépendante de $s$, on a bien montré que l'application $s \mapsto \mathbf{n}_{s}$ est constante.

Ecrivons maintenant la matrice $\mathbf{m}_{s}^{\prime}$ par rapport à la base $\mathscr{B}$ de $\mathfrak{g}$ sous la forme

$$
\mathbf{m}_{s}^{\prime}=\left(\begin{array}{c|c}
\mathbf{m}_{1}^{\prime}(s) & \mathbf{m}_{2}^{\prime}(s) \\
\hline{ }^{t} \mathbf{m}_{2}^{\prime}(s) & \mathbf{m}_{3}^{\prime}(s)
\end{array}\right)
$$

où $\mathbf{m}_{1}^{\prime}(s)$ (resp. $\mathbf{m}_{3}^{\prime}(s)$ ) est une matrice carrée d'ordre 4 (resp. 2) et où $\mathbf{m}_{2}^{\prime}(s)$ est une matrice à 2 colonnes et 4 lignes. Soit $\psi$ l'automorphisme de $G$ tel que la matrice de $\psi_{*}$ par rapport à la base $\mathscr{B}$ soit

$$
\left(\begin{array}{c|c}
1 & 0 \\
\hline-\mathbf{m}_{3}^{\prime-1}(s)^{t} \mathbf{m}_{2}(s) & 1
\end{array}\right)
$$

Remarquons que $\psi$ est indépendant de $s$, puisque les matrices $\mathbf{m}_{2}^{\prime}(s)$ et $\mathbf{m}_{3}^{\prime}(s)$ 
le sont. Alors la matrice de $\mathbf{m}_{s}^{\prime \prime}=\varphi^{*} \mathbf{m}_{s}^{\prime}$ par rapport à la bse $\mathscr{B}$ est

$$
\left(\begin{array}{c|c}
\mathbf{m}_{1}^{\prime \prime}(s) & 0 \\
\hline 0 & \mathbf{m}_{3}^{\prime}(s)
\end{array}\right)
$$

avec $\mathbf{m}_{1}^{\prime \prime}(s)=\mathbf{m}_{1}^{\prime}(s)-\mathbf{m}_{2}^{\prime}(s) \mathbf{m}_{3}^{\prime}(s)^{-1}{ }^{1} \mathbf{m}_{2}^{\prime}(s)$. Donc, comme dans le cas de $G_{n, p}$, on a une submersion riemannienne à fibres totalement géodésiques:

$$
\begin{aligned}
\left(\psi^{-1}(\Gamma) \backslash G, \mathbf{m}^{\prime \prime}(s)\right) & \stackrel{\pi}{\rightarrow}\left(\mathbb{R}^{4} / \mathbb{Z}^{4}, \mathbf{m}_{1}^{\prime \prime}(s)\right) \\
\sigma(x, y, z) & \mapsto(x, y)
\end{aligned}
$$

dont la base est un tore plat.

Par le même raisonnement que pour $G_{n, p}$, on montre que l'aplication $s \mapsto$ $\mathbf{m}_{1}^{\prime \prime}(s)$ est constante. Comme il en est de même pour les applications $s \mapsto \mathbf{m}_{2}^{\prime}(s)$ et $s \mapsto \mathbf{m}_{3}^{\prime}(s)$, on en déduit que pour tout $s \in I, \mathbf{m}_{1}^{\prime}(s)=\mathbf{m}_{1}^{\prime}\left(s_{0}\right)$, ce qui montre bien que l'application $s \mapsto \mathbf{m}^{\prime}(s)$ est constante.

\section{BIBLIOGRAPHIE}

1. L. Bérard-Bergery and J. P. Bourguignon, Laplacians and Riemannian submersions with totally geodesic fibers, Illinois J. Math. 26 (1982), 181-200.

2. M. Berger, P. Gauduchon, and $\mathrm{E}$. Mazet, Le spectre d'une variété riemannienne, Lecture Notes in Math., vol. 194, Springer-Verlag, Berlin and New York, 1971.

3. D. De Turck, H. Gluck, C. Gordon, and D. Webb, You cannot hear the mass of a homology class, Comment Math. Helv. 64 (1989), 589-617.

4. D. De Turck and C. Gordon, Isospectral deformations. I: Riemannian structures on two-step nilspaces, Comm. Pure Appl. Math. 42 (1989), 1067-1095.

5. C. Gordon, The Laplace spectrum versus the length spectra of Riemannian manifolds, Nonlinear Problems in Geometry (D. M. De Turck, ed.), Contemp. Math., vol. 51, Amer. Math. Soc., Providence, R.I., 1986, pp. 63-80.

6. C. Gordon and E. Wilson, Isospectral deformations of compact solvmanifolds, J. Differential Geom. 19 (1984), 245-256.

7. J. 33 (1986), 253-271.

8. F. D. Greenleaf and L. Corwin, Representation of nilpotent Lie groups and their applications. 1: Basic theory and examples, Cambridge Stud. in Adv. Math., vol. 18, 1989.

9. A. Ikeda, Isospectral problem for spherical space forms, Spectra of Riemannian Manifolds (M. Berger, S. Murakami, and T. Ochiai, eds.), Kaigai Publication, 1983, pp. 57-63.

10. Y. Kitaoka, Positive definite quadratic form with same representation number, Arch. Math. 28 (1977), 495-497.

11. A. I. Mal'cev On a class of homogeneous space, Amer. Math. Soc. Transl. (1) 9 (1962), 276-307.

12. J. Milnor, Eigenvalues of the Laplace operator on certain manifolds, Proc. Nat. Acad. Sci. U.S.A. 51 (1964), 542.

13. C. C. Moore, Representations of solvable and nilpotent groups and harmonic analysis on nil and solvmanifolds, Harmonic Analysis on Homogeneous Spaces (Williamstown, Mass., 1972), Proc. Sympos. Pure Math., vol. 26, Amer. Math. Soc., Providence, R.I., 1973, pp. 3-44.

14. __, Decomposition of unitary representations defined by discrete subgroups of nilpotent groups, Ann. of Math. (2) 82 (1965), 146-182.

15. H. Ouyang, Ph.D. Thesis, Washington University, St. Louis, 1991.

16. H. Pesce, Finitude spectrale des variétés de Heisenberg, Ann. Sci. École Norm. Sup. 25 (1992), 515-538. 
17. L. Pukansky, Leçons sur les représentations de groupe, Dunod, Paris, 1966.

18. M. S. Raghunathan, Discrete subgroups of Lie groups, Springer-Verlag, New York, 1972.

19. T. Sunada, Riemannian covering and isospectral manifolds, Ann. of Math. (2) 121 (1985), 169-186.

20. M. F. Vigneras, Variétés riemanniennes isospectrales et non isométriques, Ann. of Math. (2) 112 (1980), 21-32.

21. S. Wolpert, The eigenvalues spectrum as moduli for flat tori, Trans. Amer. Math. Soc. 244 (1978), 313-321.

Institut Fourier, B.P.74, 38402 St. Martin D'Hères Cedex, France

E-mail address: pesce@grenet.fr 